\title{
A global study of hot flow anomalies using Cluster multi-spacecraft measurements
}

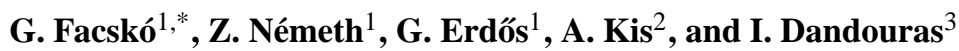 \\ ${ }^{1}$ KFKI Research Institute for Particle and Nuclear Physics, Budapest, Hungary \\ ${ }^{2}$ Geodetic and Geophysical Research Institute, Sopron, Hungary \\ ${ }^{3}$ CERS, CNRS, Toulouse, France \\ * now at: LPCE, CNRS, Orléans, France
}

Received: 17 March 2008 - Revised: 14 April 2009 - Accepted: 15 April 2009 - Published: 5 May 2009

\begin{abstract}
Hot flow anomalies (HFAs) are studied using observations of the magnetometer and the plasma instrument aboard the four Cluster spacecraft. We study several specific features of tangential discontinuities on the basis of Cluster measurements from the time periods of FebruaryApril 2003, December 2005-April 2006 and January-April 2007, when the separation distance of spacecraft was large. The previously discovered condition (Facskó et al., 2008) for forming HFAs is confirmed, i.e. that the solar wind speed and fast magnetosonic Mach number values are higher than average. Furthermore, this constraint is independent of the Schwartz et al. (2000)'s condition for HFA formation. The existence of this new condition is confirmed by simultaneous ACE magnetic field and solar wind plasma observations at the L1 point, at 1.4 million $\mathrm{km}$ distance from the Earth. The temperature, particle density and pressure parameters observed at the time of HFA formation are also studied and compared to average values of the solar wind plasma. The size of the region affected by the HFA was estimated by using two different methods. We found that the size is mainly influenced by the magnetic shear and the angle between the discontinuity normal and the Sun-Earth direction. The size grows with the shear and (up to a certain point) with the angle as well. After that point it starts decreasing. The results are compared with the outcome of recent hybrid simulations.
\end{abstract}

Keywords. Interplanetary physics (Discontinuities; Planetary bow shocks; Solar wind plasma) - Magnetospheric physics (Solar wind-magnetosphere interactions)

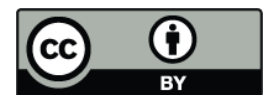

Correspondence to: G. Facskó (gfacsko@cnrs-orleans.fr)

\section{Introduction}

Although hot flow anomalies (HFAs), explosive events near the Earth's bow shock have been known more than 20 years (Schwartz et al., 1985; Thomsen et al., 1986), their theoretical explanation needs further studies (Burgess and Schwartz, 1988; Thomas et al., 1991; Lin, 2002). The most reliable description of HFAs is so far based on hybrid plasma simulations where electrons are considered as a massless and neutralizing fluid. The original motivation of this work was to verify several predictions presented in Lin (2002), but this study led us much further than we expected. In order to do this we determined the size-angle plot (described in the following section). We calculated the related angles and estimated the size in two different ways. Lin's hybrid simulation (Lin, 2002) uses a larger simulation box than in other studies mentioned above, and inserts a zero-resistivity surface (magnetopause) to the super-Alfvénic plasma flow when the simulation is initialized. This plasma flow moves parallel to the $\mathrm{x}$-axis of the box and a shock is formed. A tangential discontinuity is created ahead of the shock, and then the angle between flow direction and normal vector $(\gamma)$ can be changed. The simulations were run using different angles and their results suggested that average radius of HFAs is approximately $1-3 R_{\text {Earth }}$. A prediction of her theory is that the size of HFAs increases monotonically with $\gamma$ until $80^{\circ}$ and then begins to decrease. Another prediction is that the size of HFAs is a monotonically increasing function of the magnetic field vector direction change angle $(\Delta \Phi)$ across the discontinuity (Lin, 2002). The goal of this study was to check the validity of these predictions based on simulation results.

The four spacecraft Cluster mission provides an excellent opportunity to study HFAs (Lucek et al., 2004; Kecskeméty et al., 2006). We have identified 124 HFAs in the Cluster dataset, which enables a statistical survey. This expands the

Published by Copernicus Publications on behalf of the European Geosciences Union. 


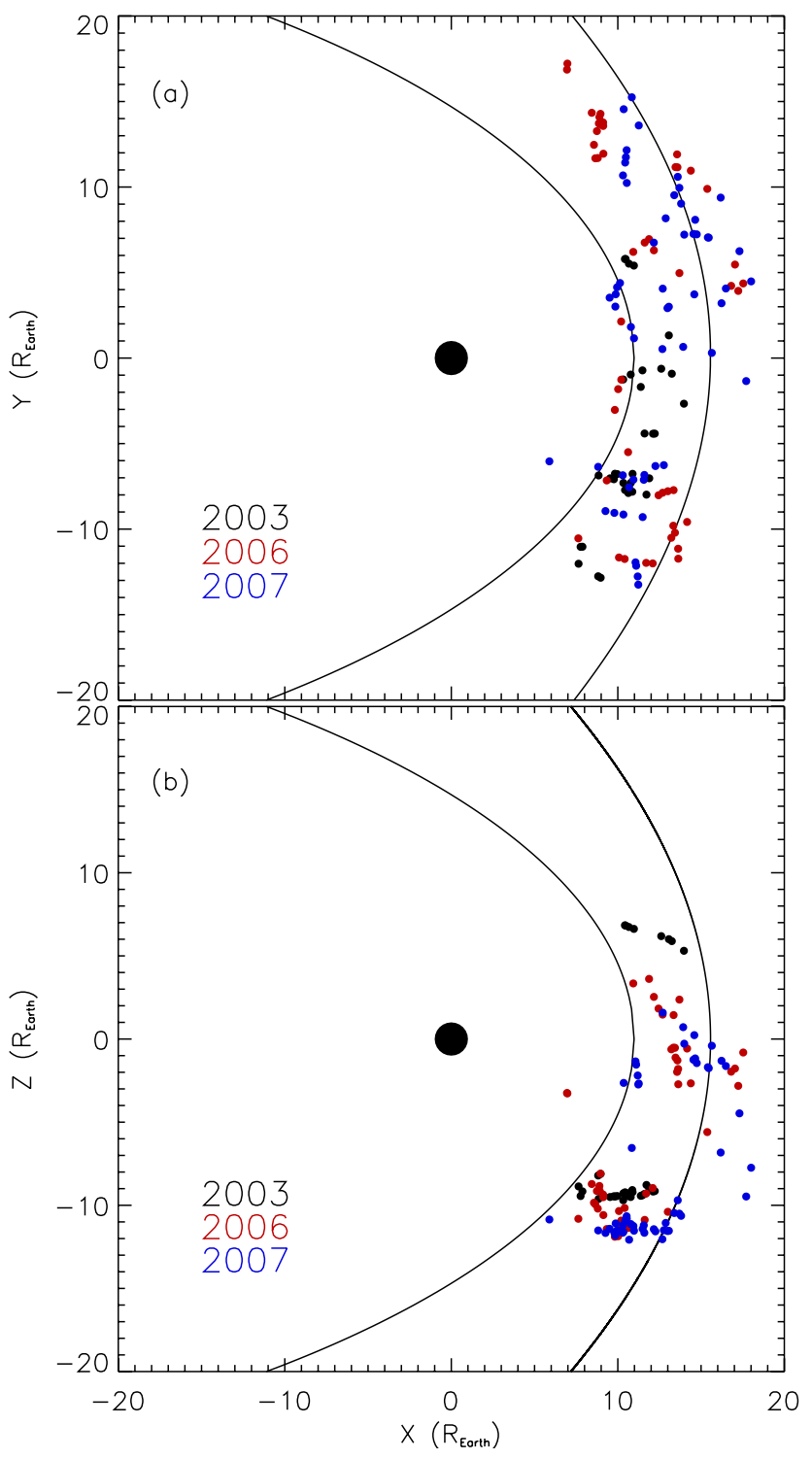

Fig. 1. HFA locations (a) in XY GSE and (b) XZ GSE plane projections and the average bow shock and magnetopause positions. The coordinates were plotted in units of $R_{\text {Earth }}$. The shapes of the magnetopause and the bow shock were calculated with the average solar wind pressure (Sibeck et al., 1991; Tsyganenko, 1995) and Alfvén-Mach number during HFA formation (Peredo et al., 1995). The black, red and blue points show Cluster positions when HFAs were observed in 2003, 2006 and 2007, respectively.

database of known events since previous analysis was based on significantly fewer events (Schwartz et al., 2000). Our results confirm the results of Lin (2002) that the size depends on the shear and on the angle between the discontinuity normal and Sun-Earth direction as well; furthermore these results strongly support the recently suggested new condition of HFA formation namely that during HFA formation the typical value of the solar wind speed is higher than the average
(Facskó et al., 2008). We have used part of Schwartz et al. (2000)'s calculations so we have checked his formula (Eq. 2) too. Finally the original purpose led us to confirm the findings of three different previous theories and to discover several new independent condition of HFA formation.

The structure of this paper is as follows: we first describe the observational methods and the observed events in Sects. 2 and 3, discuss and present our analysis methods in Sect. 4, and explain and summarize the result of our study in Sect. 5 .

\section{Data sets}

For our study we used $1 \mathrm{~s}$ and $(22.5 \mathrm{~Hz})^{-1}$ temporal resolution Cluster FGM (Fluxgate Magnetometer) magnetic field data (Balogh et al., 2001) and spin averaged time resolution CIS (Cluster Ion Spectrometry) HIA (Hot Ion Analyzer) plasma measurement data (Rème et al., 2001). We often found the magnetic signatures of the TD - which interacts with the bow shock and generates the HFA later - in ACE (Advanced Composition Explorer) MAG (Magnetometer Instrument) $16 \mathrm{~s}$ temporal resolution magnetic field data series (Smith et al., 1998). Alfvén Mach numbers were calculated and solar wind velocity was determined based on ACE SWEPAM (Solar Wind Electron, Proton, and Alpha Monitor) $16 \mathrm{~s}$ temporal resolution data (McComas et al., 1998). ACE SWEPAM data series were used instead of Cluster CIS HIA prime parameter data because in the case of very cold plasmas, as in the solar wind, where thermal velocities are very small compared to the plasma bulk velocity and to the instrument intrinsic energy (and thus velocity) resolution, the relative error in temperature can be large (Rème et al., 2001; CIS Team, 1997-present); furthermore not all the necessary CIS HIA data has been uploaded onto the Cluster Active Archive yet.

We set a series of criteria for the selection of HFA events based on Thomsen et al. (1986, 1993); Sibeck et al. (1999, 2002) that were:

1. The rim of the cavity must be visible as a sudden increase of magnetic field magnitude compared to the unperturbed solar wind region's value. Inside the cavity the magnetic field strength drops and its direction turns around.

2. The solar wind speed drops and its direction always turns away from the Sun-Earth direction.

3. The solar wind temperature increases and its value reaches up to several ten million Kelvin degrees.

4. The solar wind particle density also increases on the rim of the cavity and drops inside the HFA.

Using these criteria we identified 124 events in the 2003, 2006 and 2007 data. Two of these events were studied by Kecskeméty et al. (2006), and a statistical study of 33 events 
Table 1. The list of studied HFA events and spacecraft positions where HFA was observed in GSE system, in $R_{\text {Earth }}$ units. An empty cell indicates that the satellite in question did not observe the magnetic signature of a HFA.

\begin{tabular}{|c|c|c|c|c|c|c|c|c|c|c|}
\hline \multirow{2}{*}{$\begin{array}{c}\text { date } \\
\text { (yymmdd) }\end{array}$} & \multirow{2}{*}{$\begin{array}{l}\text { time } \\
\text { (UT) }\end{array}$} & \multicolumn{9}{|c|}{$\mathrm{s} / \mathrm{c}$ positions } \\
\hline & & & $\mathrm{C} 1$ & & $\mathrm{C} 2$ & & & $\mathrm{C} 3$ & & $\mathrm{C} 4$ \\
\hline 030216 & 10:04 & 10.57, & $-1.19,-9.57$ & 11.25 & -0.55 & -9.43 & 11.95, & $-0.67,-9.58$ & 12.14, & $-0.47,-9.00$ \\
\hline 030216 & & & $-1.45,-9.66$ & & & & 11.27, & $-0.90,-9.69$ & 11.46, & $-0.73,-9.12$ \\
\hline 030216 & 11:00 & 9.58 & $-1.53, \quad 9.67$ & & & & 11.06, & $-0.98,-9.72$ & & \\
\hline 030217 & $9: 59$ & 10.32, & 5.78 & 9.59 & 10.77, & 6.93 & & & 10.91, & 5.70 \\
\hline 030217 & :05 & 10.43, & 5.79 & 10.90, & 5.10 & 6.88 & & & 11.03, & 5.71, \\
\hline 030217 & 10:07 & 10.47, & $5.79, \quad 6.82$ & & & & & & & \\
\hline 030221 & 04:18 & 10.43, & $-2.08,-9.60$ & 11.17, & -1.49 & -9.46 & 11.85 & $-1.67,-9.62$ & 12.06, & $-1.50,-9.03$ \\
\hline 030307 & 12 & 11.29, & $-4.56,-9.35$ & 12.08 & -4.21 & & 12.62 & $-4.51,-9.33$ & 12.89 & $-4.37,-8.73$ \\
\hline 030307 & & 11.18, & $-4.57,-9.38$ & 11.98, & -4.22 & -9.19 & 12.52 & $-4.52,-9.36$ & 12.78, & $-4.38,-8.76$ \\
\hline 0303 & $10: 15$ & & & 11.1 & -4.23 & -9.41 & 11.71, & $-4.56,-9.56$ & 11.97, & $-4.44,-8.98$ \\
\hline 030308 & :07 & 12.89, & 1.71, & 13.07, & 0.92 & 6.30 & 12.90, & $1.21, \quad 5.69$ & 13.40, & $1.46, \quad 5.80$ \\
\hline 030317 & & 12.51, & $-0.22, \quad 6.42$ & 12.55 & -1.03 & 6.48 & 12.41, & $-0.70, \quad 5.88$ & 12.95, & -0.55 \\
\hline & & 13.14, & $-0.51, \quad 6.11$ & 3.18 & -1.32 & 6.18 & 13.07, & $-1.00, \quad 5.57$ & 13.58, & $-0.83, \quad 5.68$ \\
\hline & & 10.47, & $-6.86,-9.31$ & 11.30, & -6.68 & -9.1 & 11.74, & $-7.07,-9.28$ & 12.03, & $-6.98,-8.68$ \\
\hline 0 & & 9.96, & $-6.78,-9.44$ & & & & & & & \\
\hline 030319 & & 9.83 & $-6.76,-9.47$ & & & & & & & \\
\hline & & 10.33, & $-7.30,-9.28$ & & & & & & & \\
\hline & & & $-7.21,-9.41$ & 10.70, & -7.06 & -9.22 & 11.14, & $-7.49,-9.38$ & 11.44, & $-7.42,-8.79$ \\
\hline & & & $-6.99,-9$. & 9.67 & -6 & & 10.17, & $-7.29,-9$ & & $-7.24,-$ \\
\hline 030321 & & 8.52 & $-6.93,-9.68$ & 9.44 & & & 9.96 & $-7.25,-9.68$ & 10.22 & $-7.19,-9.10$ \\
\hline 030321 & 7:56 & 7.79, & $-6.75,-9.78$ & 8.75 & -6.58 & -9 & 9.30 & $-7.09,-9.80$ & 9.55 & $-7.04,-9.23$ \\
\hline & 19: & & & 13.84, & -2 & & 13.79 & $-2.62, \quad 5.05$ & 14.28 & $-2.47, \quad 5.19$ \\
\hline 0 & & 10.86, & $-7.87,-9.01$ & 11.66, & -7.7 & -8 & 12.02, & $-8.17,-8$. & 12.34, & $-8.10,-8.36$ \\
\hline 0 & & & $-7.70,-9.30$ & & & -9 & 11.21, & $-8.02,-9.27$ & 11.51, & $-7.95,-8.67$ \\
\hline 030324 & & 9.50 & $-7.59,-9.43$ & 10.36, & -7.48 & -9.24 & 10.79, & $-7.93,-9.40$ & 11.08, & $-7.87,-8.81$ \\
\hline 030324 & 01:08 & & & & & & 10.63, & $-7.89,-9.45$ & & \\
\hline & & & & & & & 7.76 & $4,-9.44$ & & \\
\hline & & & & & & & $7.73,-$ & $-11.01,-9.45$ & $8.02,-$ & $-11.05,-8.87$ \\
\hline 030416 & & $8.32,-$ & $-12.45,-8.35$ & $8.96,-$ & -12.69 & -8.09 & $9.12,-$ & $-13.10,-8.31$ & $9.47,-$ & $-13.13,-7.71$ \\
\hline 030416 & 6.23 & & $-12.36,-8.44$ & $8.81,-$ & & -8.19 & $8.98,-$ & $-13.02,-8.40$ & $9.32,-$ & $-13.05,-7.80$ \\
\hline & & & -9.09 & & & & $7.85,-$ & $-12.33,-9$ & $8.17,-$ & $-12.37,-8.45$ \\
\hline & & 6.15 , & $3,-3.18$ & 7.28 & 17 & -2 . & 7.39, & $16.48,-4.19$ & 6.97, & $16.49,-3.29$ \\
\hline & & 6.42 & $17.31,-3.69$ & 7.52 & & & & & & \\
\hline & & 8.41 , & $14.50,-8.60$ & 8.95, & 14.42 & -7 & 9.14 & $13.38,-9.69$ & 9.08 & $14.07,-9.09$ \\
\hline & & 8.43 , & $14.34,-8.72$ & 8.96, & 14.28 & & 9.14, & $13.22,-9.81$ & 9.10 & $13.93,-9.22$ \\
\hline & & & $2,-8.89$ & & & & 9.15 & 12. & 9.12 & $13.77,-9.35$ \\
\hline 0 & 2 & 8.47, & $14.08,-8.92$ & 8.96, & 14.00, & -8.32 & 9.15 & $12.94,-10.00$ & 9.13, & $13.68,-9.43$ \\
\hline & & 8.55 & $13.30,-9.41$ & 8.94, & 13.24 & -8.87 & & & & \\
\hline & & 8.57, & $12.47,-9.85$ & & & & & & & \\
\hline & & 8.56 & $12.09,-10.01$ & 8.82 & 12.04, & -9.54 & 9.02 & $10.96,-11.04$ & 9.13, & $11.95,-10.59$ \\
\hline & & & & & & & & & & \\
\hline 60117 & & 11.50, & $7.12,-10$ & 11.60, & 7.09 & -10.16 & 11.42, & $5.96,-11.53$ & 11.93, & $6.83,-11.26$ \\
\hline & & 10.25, & $2.38,-11.00$ & 10.09, & & & 9.77 & $1.44,-11$ & 10.69, & $2.28,-11.84$ \\
\hline 060128 & 5:56 & 12.97, & $12.00,-1.09$ & 13.92, & 11.18, & -0.17 & 13.78, & $10.71,-2.05$ & 13.19, & $10.76,-1.16$ \\
\hline 060128 & $06: 12$ & 13.09, & $12.00,-1.26$ & 14.03, & 11.18, & -0.34 & 13.88, & $10.69,-2.22$ & 13.32, & $10.75,-1.34$ \\
\hline & & 13.56, & $11.91,-1.98$ & & & & & & & \\
\hline & & & $11.79,-2.59$ & & & & 14.62, & $10.42,-3$ & 14.20 & $10.60,-2$ \\
\hline & & & $10.67,-5.43$ & & & & 15.44 & $9.23,-6.56$ & 15.33, & $9.61,-5.80$ \\
\hline 060214 & $22: 33$ & 10.23, & $-1.38,-11.02$ & 10.18 & $-1.15,-$ & -10.82 & 9.46 & $-2.05,-11.84$ & 10.58 & $-1.59,-11.88$ \\
\hline
\end{tabular}


Table 1. Continued.

\begin{tabular}{|c|c|c|c|c|c|c|c|c|c|c|}
\hline \multirow{2}{*}{$\begin{array}{c}\text { date } \\
\text { (yymmdd) }\end{array}$} & \multirow{2}{*}{$\begin{array}{l}\text { time } \\
\text { (UT) }\end{array}$} & \multicolumn{9}{|c|}{$\mathrm{s} / \mathrm{c}$ positions } \\
\hline & & & $\mathrm{C} 1$ & & $\mathrm{C} 2$ & & & $\mathrm{C} 3$ & & $\mathrm{C} 4$ \\
\hline 060215 & $23: 29$ & 11.55, & $7.51, \quad 3.19$ & 12.20, & 6.40 & 4.04 & 12.17, & $6.30, \quad 2.53$ & 10.93, & $6.21, \quad 3.34$ \\
\hline 060221 & 01:50 & 17.04, & $5.47,-1.77$ & 17.53, & 4.36, & -0.81 & 17.23, & $3.93,-2.82$ & 16.81, & $4.22,-1.96$ \\
\hline 060222 & 01:09 & & & & & & 9.82 & $-3.03,-11.89$ & & \\
\hline 060223 & 04:14 & 13.62, & $5.91, \quad 2.30$ & 14.12, & 4.71, & 3.20 & 14.03, & $4.56, \quad 1.58$ & 13.02 & 4.69, \\
\hline 060310 & $15: 31$ & 10.84 & $-5.30,-11.01$ & 10.97, & $-5.22,-$ & & 9.83 & $-5.76,-11.91$ & 10.84 & $-5.72,-11.82$ \\
\hline 060320 & 04:16 & 9.58 & $-7.01,-11.07$ & 9.75 & $-6.93,-$ & -10.80 & 8.51, & $-7.27,-11.95$ & 9.49 & $-7.41,-11.89$ \\
\hline 060322 & 00 & 13.35, & $-7.40,-10.08$ & 13.47, & -7.71 & -9.55 & 12.23 & $-8.02,-11.14$ & 12.96, & $-8.01,-10.80$ \\
\hline 060410 & :37 & $2.65,-$ & $-11.59,-8.68$ & $12.54,-$ & -12.17 & -7.96 & $11.34,-$ & $-12.11,-9.83$ & $11.85,-$ & $-12.15,-9.37$ \\
\hline 060410 & 05:27 & 12.23, & $-11.59,-9.01$ & $12.16,-$ & -12.12 & -8.32 & $10.93,-$ & $-12.05,-10.14$ & $11.48,-$ & $-12.13,-9.71$ \\
\hline 060410 & 07:53 & 10.89 & $-11.46,-9.85$ & $10.90,-$ & -11.85 & & $9.61,-$ & $-11.75,-10.94$ & $10.23,-$ & $-11.92,-10.59$ \\
\hline 060410 & $3: 28$ & 10.54 & $-11.40,-10.03$ & $10.57,-$ & -11.75 & -9.47 & $9.26,-$ & $-11.64,-11.10$ & $9.91,-$ & \\
\hline 060410 & 52 & $7.54,-$ & $-10.49,-10.98$ & $7.72,-$ & -10.59 & -10.64 & & & & \\
\hline 060416 & 7.39 & 13.07, & $\begin{array}{ll}-7.25, & 1.87\end{array}$ & 12.31, & -8.49 & 1.06 & & & & \\
\hline 060416 & $12: 45$ & 11 & $-7.33, \quad 1.81$ & 12.40 & -8.37 & 2.81 & 12.34 & $\begin{array}{ll}-8.57, & 0.99\end{array}$ & 11.92, & $\begin{array}{ll}-7.82, & 1.71\end{array}$ \\
\hline 060416 & $13: 22$ & 13.34, & $\begin{array}{ll}-7.72, & 1.44\end{array}$ & 14.04 & -9.21 & -0.12 & $13.07,-$ & $-10.38,-1.08$ & 12.88 & $-9.82,-0.36$ \\
\hline 50416 & & 14.14 & $-9.51,-0.48$ & $13.46,-$ & -10.54 & 0.57 & $13.14,-$ & $-10.68,-1.47$ & $12.99,-$ & $-10.13,-0.74$ \\
\hline 060416 & & 14.16, & $-9.58,-0.57$ & $13.48,-$ & -10.59 & 0.50 & $13.16,-$ & $-10.73,-1.54$ & $13.01,-$ & $-10.19,-0.82$ \\
\hline 060416 & $18: 32$ & $14.34,-$ & $-10.46,-1.71$ & $13.70,-$ & -11.46 & -0.66 & $13.25,-$ & $-11.56,-2.77$ & $13.21,-$ & $-11.11,-2.06$ \\
\hline 060416 & 20:01 & 14.35 & $-11.06,-2.60$ & $13.75,-$ & -12.04 & -1.56 & $13.19,-$ & $-12.10,-3.70$ & $13.23,-$ & $-11.72,-3.01$ \\
\hline 070104 & & & 12. & & & -9.78 & 10.69, & & & \\
\hline 070104 & 4:36 & 10.13, & 12. & 10.5 & 12.34, & -9.99 & 10.64, & $11.25,-11.40$ & 10.64, & $11.31,-11.38$ \\
\hline 07 & & & & & 11.3 & -10 & 10.44 & & 10.45 & \\
\hline 070104 & & 10.10 & $11.74,-10.69$ & 10.48, & $12.03,-$ & -10.14 & 10.59 & & 10.59 & \\
\hline 070106 & $16: 07$ & 10.39, & $10.06,-11.01$ & 10.69, & $10.41,-$ & -10.60 & & & & \\
\hline 070 & & & 15. & 11.03, & 15.5 & & 11.08, & $14.82,-7.27$ & 11.06, & $14.84,-7.22$ \\
\hline 070116 & $09: 4$ & 10.08, & -11 & 10.1 & & -11 & 10.11, & 3. & 10.1 & \\
\hline 07 & & & & 10. & & -11 & 9.93 & & 9.97, & \\
\hline 070116 & & 9.48 & $3.63,-11.04$ & & $4.15,-$ & -11.08 & 9.48 & $3.14,-11.77$ & 9.53 & $3.21,-11.78$ \\
\hline 070117 & $16: 38$ & 10.35 & $14.55,-2.64$ & 11.24, & 13.79, & & 11.28, & $13.50,-3.37$ & 11.24, & $13.50,-3.317$ \\
\hline & & & 10 & & & & & & & 0.50 \\
\hline 07 & 0 & .09 & 10. & 9 & 10.0 & -9 & & & & \\
\hline 070118 & & 12.65 & & 13.05, & 8.7 & & & $7.66,-11.61$ & 12.91, & $7.71,-11.59$ \\
\hline 070118 & $14: 35$ & 12.01, & $7.08,-11.12$ & 12.31, & $7.34,-$ & -10.79 & 12.15, & $6.26,-11.93$ & 12.18, & $6.32,-11.91$ \\
\hline 070118 & & & & & $3.98,-$ & -11 & 9.83 & $2.98,-1$ & 9.87 & $3.04,-11.82$ \\
\hline 07012 & $18: 1$ & 13.58, & 9.71, & 13.90, & $8.66,-$ & -10 & 13.92, & $8.71,-10.9$ & & \\
\hline 07 & $16: 4$ & & $1.63,-$ & 10.85, & 2.01 & & & & & \\
\hline 070201 & $06: 48$ & 15.78, & $10.20,-6.63$ & 16.48 & 9.54, & -5.61 & 16.23, & $8.88,-7.56$ & 16.22 & $8.91,-7.51$ \\
\hline 070201 & 22:07 & 13.05, & $3.36,-11.2$ & 13.32, & $3.52,-$ & & 12.92 & $2.53,-12$ & 12.97, & $2.59,-11.99$ \\
\hline & & 12.97, & & 13.2 & & & & & & $2.51,-12.00$ \\
\hline 070202 & 01:31 & 11.02, & $1.36,-11.17$ & 11.17, & $1.71,-$ & -11.14 & 10.82, & $0.75,-11.90$ & 10.88, & $0.80,-11.90$ \\
\hline 070209 & $02: 14$ & & & & & & 12.68, & $0.53,-12.04$ & & \\
\hline 070215 & 01:35 & 13.61, & $8.15,-0.21$ & 14.10, & 6.94 & 0.93 & 14.16, & $6.88,-0.96$ & 14.10, & $6.89,-0.89$ \\
\hline 070215 & $02: 29$ & 14.21, & $8.13,-0.74$ & & & & & $6.82,-1.53$ & & $6.83,-1.46$ \\
\hline & $02: 49$ & 14.41, & $8.11,-0.94$ & & & & 14.93, & $6.79,-1.73$ & 14.88 & $6.80,-1.67$ \\
\hline & & 14.65 & $8.08,-1.17$ & & & & & & & \\
\hline 0215 & & 15.05 & $8.02,-1.58$ & 15.56, & 6.84 & -0 & 15.5 & $6.67,-2$. & 15.4 & $6.68,-2.35$ \\
\hline 070215 & 04:01 & 15.10, & $8.01,-1.64$ & 15.62, & 6.83 & -0.48 & 15.58, & $6.65,-2.47$ & 15.54, & $6.67,-2.41$ \\
\hline 070215 & 08:44 & 17.02 & $7.22,-4.29$ & 17.57, & 6.18 & -3.20 & 17.33, & $5.79,-5.23$ & 17.31, & $5.81,-5.17$ \\
\hline 070215 & $15: 16$ & 17.86, & $5.35,-7.49$ & 18.36, & 4.60 & -6.59 & 17.89, & $3.97,-8.47$ & 17.90, & $4.01,-8.42$ \\
\hline
\end{tabular}


Table 1. Continued.

\begin{tabular}{|c|c|c|c|c|c|c|c|c|c|c|}
\hline \multirow{2}{*}{$\begin{array}{c}\text { date } \\
\text { (yymmdd) }\end{array}$} & \multirow{2}{*}{$\begin{array}{l}\text { time } \\
\text { (UT) }\end{array}$} & \multicolumn{9}{|c|}{ s/c positions } \\
\hline & & & $\mathrm{C} 1$ & & $\mathrm{C} 2$ & & & C3 & & $\mathrm{C} 4$ \\
\hline 070301 & $04: 56$ & 12.47, & 5.00 & 12.64, & 3.71 , & 2.67 & 12.88, & 3.76 & 12.80 & 3.77 \\
\hline 070301 & $07: 10$ & 14.38, & 4.72 & 14.61, & 3.40 & 1.40 & 14.72, & $3.37,-0.44$ & 14.65 , & $3.39,-0.37$ \\
\hline 070301 & $09: 43$ & 16.06, & $4.24,-1.16$ & 16.32, & 2.93 & -0.08 & 16.29, & $2.81,-2.03$ & 16.24, & $2.83,-1.96$ \\
\hline 070301 & $10: 30$ & 16.49 & $4.07,-1.63$ & & & & & & & \\
\hline 070302 & 02:03 & 17.87, & $-0.58,-9.15$ & 18.12, & -1.23 & -8.49 & 17.40, & $-1.80,-10.15$ & 17.44, & $-1.77,-10.10$ \\
\hline 070313 & $05: 36$ & 15.61, & $1.36,-0.24$ & 15.65, & -0.02 & 0.77 & 15.68, & $-0.09,-1.11$ & 15.63, & $-0.05,-1.04$ \\
\hline 070314 & $07: 53$ & 13.15 & $-5.91,-11.14$ & 13.21, & $-6.06,-$ & -10.90 & 12.31, & $-6.54,-11.98$ & 12.38, & $-6.53,-11.97$ \\
\hline 070314 & $08: 36$ & 12.64 & $-6.00,-11.21$ & 12.70 & -6.10 & -11.00 & 11.81, & $-6.58,-12.03$ & 11.88 & $-6.57,-12.02$ \\
\hline 070314 & $12: 51$ & 9.18 & $-6.27,-11.17$ & 9.21 & $-6.06,-$ & -11.17 & 8.40 & $-6.56,-11.85$ & 8.49 & $-6.56,-11.86$ \\
\hline 070314 & $15: 52$ & 6.20 & $-6.10,-10.53$ & 6.20 & $-5.68,-$ & -10.67 & 5.52 & $-6.18,-11.09$ & 5.63 & $-6.19,-11.12$ \\
\hline 070315 & $12: 14$ & 13.84 & $1.56, \quad 1.21$ & & & & 14.01, & $0.18, \quad 0.43$ & 13.93, & $0.22, \quad 0.50$ \\
\hline 070316 & $18: 13$ & 12.02 & $-6.57,-11.25$ & & & & 11.16, & $-7.09,-12.06$ & & \\
\hline 070316 & $19: 56$ & 10.69 & $-6.67,-11.30$ & 10.72, & $-6.61,-$ & -11.20 & 9.85 & $-7.07,-12.05$ & 9.93 & $-7.07,-12.04$ \\
\hline 070319 & 03:39 & 11.55, & $-7.10,-11.28$ & 11.57, & $-7.14,-$ & -11.12 & 10.64 & $-7.55,-12.08$ & 10.72 & $-7.55,-12.07$ \\
\hline 070319 & $04: 27$ & 10.93, & $-7.12,-11.31$ & 10.95, & -7.10 & -11.19 & & & & \\
\hline 070328 & $13: 41$ & 12.01, & $-9.01,-11.06$ & 11.94, & $-9.23,-$ & -10.79 & 10.97, & $-9.47,-11.93$ & 11.05, & $-9.48,-11.91$ \\
\hline 070328 & $15: 22$ & 10.84 & $-8.95,-11.24$ & 10.78, & $-9.04,-$ & -11.06 & 9.82 & $-9.30,-12.06$ & 9.90 & $-9.31,-12.05$ \\
\hline 070328 & $16: 07$ & 10.29 & $-8.89,-11.29$ & 10.23, & $-8.92,-$ & -11.14 & 9.28 & $-9.19,-12.08$ & 9.36 & $-9.20,-12.07$ \\
\hline 070328 & $16: 50$ & 9.74 & $-8.82,-11.31$ & 9.70 & -8.80 & -11.19 & 8.75 & $-9.07,-12.08$ & 8.84 & $-9.09,-12.07$ \\
\hline 070429 & $20: 40$ & $11.79,-$ & $-11.03,-1.00$ & $10.88,-$ & -12.27 & -0.21 & 10.79 & $-12.27,-2.12$ & $10.79,-$ & $-12.21,-2.05$ \\
\hline 070429 & 21:00 & $11.84,-$ & $-11.24,-1.20$ & $10.92,-$ & -12.47 & -0.40 & $10.82,-$ & $-12.47,-2.32$ & $10.82,-$ & $-12.40,-2.24$ \\
\hline 070429 & $22: 05$ & $11.97,-$ & $-11.89,-1.83$ & $11.04,-$ & -13.01 & -1.04 & 10.88 & $-13.07,-2.99$ & $10.89,-$ & $-13.01,-2.91$ \\
\hline 070429 & 23:02 & $12.04,-$ & $-12.41,-2.37$ & 11.10 & -13.58 & -1.57 & $10.89,-$ & $-13.53,-3.54$ & $10.89,-$ & $-13.47,-3.46$ \\
\hline 070430 & 02:01 & $11.98,-$ & $-13.79,-4.03$ & & & & & & & \\
\hline
\end{tabular}

in the 2003 data was analyzed by Facskó et al. (2008). The positions of the events are given in Table 1 and Fig. 1. All of them were observed beyond the bow shock in the FebruaryApril 2003, December 2005-April 2006 and January-April 2007 time intervals. A fraction of these events was located very far from the bow shock and the Earth ( $\left.\geq 19 R_{\text {Earth }}\right)$, occurring mainly in 2007. Only the position of tetrahedron center of the Cluster SC is plotted in Figs. 1, 2 because the length of the orbital section is comparable with the thickness of the lines drawn. The bow shock position was calculated using the average Alfvén Mach number during formation of the events $\left(M_{\mathrm{A}}=11.8\right.$, Sect. 3.2$)$ according to the model described in Peredo et al. (1995). The position of the magnetopause was calculated using the same average solar wind pressure $(1.73 \pm 0.8 \mathrm{nPa}$, Sect. 3.3) as that in Sibeck et al. (1991) and Tsyganenko (1995).

The cylindrical projection of the center of the Cluster SC positions is also plotted to more easily determine whether the observations were performed beyond or inside the average bow shock (Fig. 2). Figure 1 seems to indicate that the HFAs are mostly located within the magnetosheath, with some inside the magnetosphere. However, this is only a feature of the applied projection. The position of the bow shock was calculated using the average solar wind pressure during, the HFA event. All HFAs were beyond the actual bow shock when we observed them. However the bow shock position changes quickly, presenting explanation for why some of the events seem to be located in the magnetosheath.

\section{Analysis}

\subsection{Size-angle plots}

The main purpose of this paper is to determine experimentally the role the different angles $(\gamma, \Delta \Phi)$ play in controlling HFA size. In the next two sections we therefore calculate the angles associated with each HFA and its size.

\subsubsection{Determination of angles}

The two angles (the $\gamma$ and the $\Delta \Phi$ ) mentioned before are considered to be very important in the formation of HFA events. We are able to measure these angles and thus to compare the results of measurements with the predictions of earlier simulations. Unfortunately, triangulation techniques can not be used to determine these angles because of strong magnetic field fluctuations. Thus the direction of the TD normal vector was determined by the cross-product method and minimum-variance techniques using Cluster FGM (Balogh et al., 2001) and ACE MAG measurements data (Smith et al., 1998). The temporal resolution of FGM data series were $1 \mathrm{~s}$ and MAG's resolution was $16 \mathrm{~s}$. We accepted the result of 
Table 2. Parameters of TD normal vectors: $\lambda_{2} / \lambda_{3}$ is the ratio of 2 nd and 3 rd eigenvalues, $B_{\min }$ is the smallest magnetic field component in minimum variance system, $\Delta \boldsymbol{n}$ is the error cone of minimum variance method, $\gamma$ is the angle between the Sun direction and TD normal, $\Delta \Phi$ is the direction change across the discontinuity and $\theta$ the angle between the bow shock normal and the $\boldsymbol{B}$ magnetic field vector. Boldface letter shows quasi-perpendicular conditions; the angles were calculated by scaling a model BS to the location of Cluster-1 and 3 spacecraft.

\begin{tabular}{|c|c|c|c|c|c|c|c|c|c|c|}
\hline $\begin{array}{c}\text { date } \\
\text { (yymmdd) }\end{array}$ & $\begin{array}{l}\text { time } \\
\text { (UT) }\end{array}$ & $\mathrm{s} / \mathrm{c}$ & $\boldsymbol{n}_{\boldsymbol{B}_{\mathrm{u}} \times \boldsymbol{B}_{\mathrm{d}}}$ & $\boldsymbol{n}_{\text {minvar }}$ & $\frac{\lambda_{2}}{\lambda_{3}}$ & $\begin{array}{l}B_{\min } \\
(n T)\end{array}$ & $\begin{array}{l}\Delta \boldsymbol{n} \\
\left({ }^{o}\right)\end{array}$ & $\begin{array}{c}\gamma \\
\left({ }^{o}\right)\end{array}$ & $\begin{array}{l}\Delta \Phi \\
\left({ }^{o}\right)\end{array}$ & $\begin{array}{c}\theta_{\mathrm{C} 1, \mathrm{C} 3} \\
\left({ }^{o}\right)\end{array}$ \\
\hline 030216 & 10:04 & $\mathrm{C} 1$ & $0.53,-0.70, \quad 0.47$ & $0.40,-0.69, \quad 0.61$ & 1.1 & 1.50 & 76.53 & 66 & 51 & 27,27 \\
\hline \multirow[t]{4}{*}{030216} & $10: 48$ & $\mathrm{C} 1$ & $-0.06, \quad 0.39, \quad 0.92$ & $-0.06, \quad 0.39, \quad 0.92$ & 4.0 & 0.00 & 8.18 & 93 & 73 & 37,45 \\
\hline & & $\mathrm{C} 2$ & & $-0.29, \quad 0.37, \quad 0.88$ & 1.9 & 1.50 & 13.74 & & & \\
\hline & & $\mathrm{C} 3$ & & $0.12, \quad 0.44, \quad 0.89$ & 1.6 & -0.63 & 18.65 & & & \\
\hline & & $\mathrm{C} 4$ & & $-0.11, \quad 0.41, \quad 0.91$ & 2.0 & 0.07 & 13.84 & & & \\
\hline 030216 & $11: 00$ & ACE & $-0.21,-0.98,-0.03$ & $\begin{array}{lll}0.10, & 0.98, & 0.20\end{array}$ & 2.0 & 0.86 & 30.95 & 98 & 42 & \\
\hline 030216 & 11:02 & $\mathrm{ACE}$ & $0.19,-0.09, \quad 0.98$ & $0.21,-0.08, \quad 0.97$ & 1.7 & -0.11 & 32.67 & 80 & 61 & 48,48 \\
\hline 030217 & 09:59 & ACE & $-0.46, \quad 0.18, \quad 0.87$ & $-0.63, \quad 0.23,0.74$ & 1.7 & 0.82 & 31.71 & 99 & 19 & 14,13 \\
\hline 030217 & 10:05 & ACE & $0.70, \quad 0.63,-0.33$ & $0.70, \quad 0.63,-0.33$ & 8.5 & -0.02 & 10.05 & 48 & 73 & 31,28 \\
\hline 030217 & 10:07 & ACE & $\begin{array}{lll}0.66, & 0.75, & 0.05\end{array}$ & $\begin{array}{lll}0.67, & 0.74, & 0.05\end{array}$ & 4.7 & -0.08 & 23.32 & 54 & 63 & 32,29 \\
\hline 030217 & 10:08 & ACE & $0.17, \quad 0.48,-0.86$ & $-0.06,-0.68, \quad 0.73$ & 9.8 & 1.24 & 41.98 & 82 & 53 & 31,28 \\
\hline \multirow[t]{4}{*}{030221} & 04:18 & $\mathrm{C} 1$ & & $0.71,-0.66,-0.25$ & 5.2 & -1.24 & 7.91 & & & 17,21 \\
\hline & & $\mathrm{C} 2$ & & $0.71,-0.66,-0.24$ & 3.8 & -1.18 & 9.24 & & & \\
\hline & & $\mathrm{C} 3$ & & $0.76,-0.62,-0.19$ & 4.2 & -0.80 & 8.76 & & & \\
\hline & & $\mathrm{C} 4$ & $-0.67, \quad 0.73,-0.12$ & $0.73,-0.62,-0.27$ & 5.8 & -1.08 & 7.33 & 96 & 9 & \\
\hline 030307 & 09:12 & ACE & $0.81, \quad 0.22,-0.54$ & $0.80, \quad 0.35,-0.50$ & 1.2 & -0.76 & 75.06 & 66 & 30 & 77,72 \\
\hline 030307 & 09:19 & ACE & $0.72, \quad 0.41,-0.55$ & $0.95, \quad 0.06,-0.31$ & 1.2 & 0.97 & 59.14 & 85 & 7 & 63,71 \\
\hline \multirow[t]{4}{*}{030307} & $10: 15$ & ACE & & $\begin{array}{lll}0.61, & 0.39, & 0.69\end{array}$ & 1.1 & 0.12 & 78.12 & & & 67,68 \\
\hline & & $\mathrm{C} 2$ & $-0.53,-0.43,-0.73$ & $0.22, \quad 0.75,-0.62$ & 1.8 & 0.61 & 15.55 & & & \\
\hline & & $\mathrm{C} 3$ & & $\begin{array}{lll}0.43, & 0.50, & 0.76\end{array}$ & 1.8 & 0.01 & 19.85 & & & \\
\hline & & $\mathrm{C} 4$ & & $0.17, \quad 0.79,-0.60$ & 1.5 & 0.12 & 26.17 & & & \\
\hline \multirow[t]{2}{*}{030308} & $12: 07$ & $\mathrm{ACE}$ & & $\begin{array}{lll}0.56, & 0.38, & 0.73\end{array}$ & 1.7 & 0.00 & 34.32 & & & 66,58 \\
\hline & & $\mathrm{C} 4$ & $-0.36,-0.35,-0.87$ & $\begin{array}{lll}0.54, & 0.30, & 0.78\end{array}$ & 1.8 & 0.68 & 17.86 & 111 & 87 & 30,27 \\
\hline 030317 & 23:57 & $\mathrm{C} 4$ & $0.81, \quad 0.33,-0.48$ & $0.89, \quad 0.25,-0.38$ & 4.3 & -1.13 & 10.93 & 61 & 37 & \\
\hline 030318 & $00: 41$ & ACE & $0.62, \quad 0.75, \quad 0.23$ & $\begin{array}{lll}0.51, & 0.80, & 0.32\end{array}$ & 2.3 & 1.09 & 25.83 & 67 & 40 & 26,29 \\
\hline 030319 & $06: 20$ & ACE & $0.27,-0.73, \quad 0.63$ & $0.18,-0.71, \quad 0.67$ & 1.4 & 0.38 & 44.64 & 79 & 44 & 8,16 \\
\hline 030319 & $06: 52$ & ACE & $-0.29,-0.37,-0.88$ & $\begin{array}{lll}0.38, & 0.30, & 0.87\end{array}$ & 1.3 & -0.24 & 53.73 & 95 & 19 & 34,47 \\
\hline 030319 & 07:01 & ACE & $-0.67, \quad 0.31,-0.68$ & $-0.71, \quad 0.58,-0.40$ & 5.9 & 0.06 & 12.77 & 93 & 4 & 34,47 \\
\hline 030321 & $15: 15$ & ACE & $-0.60, \quad 0.10,-0.79$ & $0.60,-0.19, \quad 0.78$ & 1.9 & -0.13 & 27.8 & 119 & 54 & 27 \\
\hline \multirow[t]{2}{*}{030321} & $15: 48$ & $\mathrm{ACE}$ & & $\begin{array}{lll}0.71, & 0.07, \quad 0.70\end{array}$ & 1.7 & -0.21 & 13.75 & & & 26,27 \\
\hline & & $\mathrm{C} 4$ & $\begin{array}{llll}0.78, & 0.27, & 0.57\end{array}$ & $\begin{array}{lll}0.78, & 0.27, & 0.57\end{array}$ & 3.1 & 0.00 & 23.62 & 51 & 54 & \\
\hline 030321 & $16: 57$ & ACE & $0.43, \quad 0.73, \quad 0.53$ & $\begin{array}{lll}0.40, & 0.76, & 0.52\end{array}$ & 2.5 & 0.12 & 24.91 & 73 & 42 & 24,22 \\
\hline \multirow[t]{3}{*}{030321} & $17: 12$ & $\mathrm{ACE}$ & & $0.55,-0.34, \quad 0.76$ & 6.0 & 0.08 & 13.22 & & & 39,35 \\
\hline & & $\mathrm{C} 3$ & $0.60,-0.29, \quad 0.75$ & $0.64,-0.29, \quad 0.71$ & 11.4 & -0.24 & 6.27 & 53 & 92 & \\
\hline & & $\mathrm{C} 4$ & & $0.58,-0.32, \quad 0.74$ & 3.9 & -0.40 & 12.08 & & & \\
\hline 030321 & $17: 56$ & ACE & $-0.13, \quad 0.19, \quad 0.97$ & $\begin{array}{lll}0.77, & 0.23, & 0.59\end{array}$ & 4.2 & -0.41 & 16.10 & 95 & 47 & 81,84 \\
\hline 030322 & $19: 58$ & $\mathrm{C} 4$ & $0.43,-0.15,-0.89$ & $-0.55, \quad 0.25, \quad 0.80$ & 1.0 & 1.16 & 87.27 & 78 & 30 & 29,32 \\
\hline \multirow[t]{3}{*}{030323} & $23: 22$ & $\mathrm{ACE}$ & & $\begin{array}{lll}0.14, & 0.86, & 0.49\end{array}$ & 2.4 & -0.09 & 19.81 & & & 19,26 \\
\hline & & $\mathrm{C} 3$ & $\begin{array}{lll}0.46, & 0.87, & 0.17\end{array}$ & $\begin{array}{lll}0.36, & 0.90, & 0.23\end{array}$ & 3.2 & 0.44 & 10.26 & 63 & 80 & \\
\hline & & $\mathrm{C} 4$ & & $\begin{array}{lll}0.32, & 0.91, & 0.24\end{array}$ & 1.9 & 0.36 & 16.23 & & & \\
\hline 030324 & $00: 25$ & ACE & $0.82,-0.42, \quad 0.40$ & $0.93,-0.34, \quad 0.12$ & 14.1 & -0.84 & 8.89 & 82 & 10 & 36,37 \\
\hline 030324 & $00: 57$ & $\mathrm{C} 2$ & $-0.83,-0.46, \quad 0.30$ & $0.83, \quad 0.47,-0.30$ & 1.2 & 0.05 & 35.48 & & & 16,17 \\
\hline 030324 & 01:08 & ACE & $-0.06, \quad 0.44,-0.90$ & $-0.10,-0.25, \quad 0.96$ & 3.8 & 0.43 & 18.05 & 93 & 107 & 19,16 \\
\hline 030412 & 01:38 & ACE & $-0.48,-0.29,-0.83$ & $0.67, \quad 0.15, \quad 0.72$ & 9.0 & -0.95 & 8.72 & 119 & 88 & 33,34 \\
\hline 030412 & 01:42 & ACE & $0.48, \quad 0.28, \quad 0.83$ & $\begin{array}{lll}0.56, & 0.20, & 0.80\end{array}$ & 3.8 & -0.52 & 17.02 & 76 & 31 & 35,46 \\
\hline 030416 & $16: 07$ & $\mathrm{ACE}$ & $-0.44,-0.52, \quad 0.73$ & $-0.05,-0.75, \quad 0.66$ & 6.9 & -1.28 & 11.34 & 112 & 123 & 18,16 \\
\hline 030416 & $16: 23$ & $\mathrm{ACE}$ & $0.23, \quad 0.18,-0.96$ & $-0.25,-0.19, \quad 0.95$ & 1.7 & 0.11 & 30.52 & 83 & 30 & 18,16 \\
\hline 030416 & $18: 18$ & ACE & $0.56, \quad 0.82,-0.12$ & $0.75, \quad 0.59,-0.29$ & 7.6 & -1.43 & 10.42 & 57 & 101 & 17,15 \\
\hline 051228 & $11: 18$ & $\mathrm{C} 1$ & $-0.82,-0.39, \quad 0.42$ & $-0.87, \quad 0.41,-0.29$ & 1.2 & 0.09 & 39.66 & 144 & 127 & 68,73 \\
\hline
\end{tabular}


Table 2. Continued.

\begin{tabular}{|c|c|c|c|c|c|c|c|c|c|c|}
\hline $\begin{array}{c}\text { date } \\
\text { (yymmdd) }\end{array}$ & $\begin{array}{l}\text { time } \\
\text { (UT) }\end{array}$ & $\mathrm{s} / \mathrm{c}$ & $\boldsymbol{n}_{\boldsymbol{B}_{\mathrm{u}} \times \boldsymbol{B}_{\mathrm{d}}}$ & $\boldsymbol{n}_{\text {minvar }}$ & $\frac{\lambda_{2}}{\lambda_{3}}$ & $\begin{array}{l}B_{\min } \\
(n T)\end{array}$ & $\begin{array}{l}\Delta \boldsymbol{n} \\
\left({ }^{o}\right)\end{array}$ & $\begin{array}{l}\gamma \\
(o)\end{array}$ & $\begin{array}{c}\Delta \Phi \\
(o)\end{array}$ & $\begin{array}{c}\theta_{\mathrm{C} 1, \mathrm{C} 3} \\
\left({ }^{o}\right)\end{array}$ \\
\hline 051228 & $21: 50$ & $\mathrm{ACE}$ & $-0.73, \quad 0.16,-0.66$ & $0.85,-0.18, \quad 0.50$ & 2.5 & 0.64 & 19.57 & 137 & 7 & 60,59 \\
\hline \multirow[t]{3}{*}{051228} & $22: 10$ & $\mathrm{C} 1$ & & $0.05, \quad 0.76,-0.65$ & 2.6 & 0.33 & 17.07 & & & 43,40 \\
\hline & $22: 10$ & $\mathrm{C} 2$ & & $0.18, \quad 0.79,-0.59$ & 1.5 & 0.92 & 30.05 & & & \\
\hline & $22: 10$ & $\mathrm{C} 3$ & $0.03, \quad 0.87,-0.49$ & $0.04, \quad 0.84,-0.54$ & 4.8 & 0.19 & 10.86 & 88 & 15 & \\
\hline \multirow[t]{4}{*}{051228} & $22: 20$ & $\mathrm{C} 1$ & & $0.23, \quad 0.82,-0.52$ & 1.9 & -0.49 & 16.48 & & & 63,53 \\
\hline & $22: 20$ & $\mathrm{C} 2$ & $-0.25,-0.85, \quad 0.46$ & $0.13, \quad 0.85,-0.51$ & 2.2 & -0.46 & 14.28 & 104 & 97 & \\
\hline & $22: 20$ & $\mathrm{C} 3$ & & $0.29, \quad 0.84,-0.46$ & 2.2 & 0.16 & 14.65 & & & \\
\hline & $22: 20$ & $\mathrm{C} 4$ & & $0.34, \quad 0.81,-0.48$ & 1.4 & 0.11 & 27.67 & & & \\
\hline 051228 & $22: 35$ & $\mathrm{ACE}$ & $-0.64,-0.75, \quad 0.19$ & $0.62, \quad 0.75,-0.22$ & 2.9 & -0.07 & 17.20 & 129 & 115 & \\
\hline 051228 & $22: 40$ & $\mathrm{ACE}$ & $0.60, \quad 0.77,-0.21$ & $0.73, \quad 0.68,-0.06$ & 2.6 & 0.93 & 19.17 & 53 & 46 & $85, \quad 5$ \\
\hline 051229 & 00:00 & $\mathrm{ACE}$ & $0.59,-0.17,-0.79$ & $0.72,-0.12,-0.69$ & 13.4 & 0.56 & 7.28 & 54 & 98 & 83,74 \\
\hline 051229 & 01:20 & $\mathrm{C} 1$ & $-0.83,-0.16, \quad 0.54$ & $0.74, \quad 0.20,-0.64$ & 2.9 & -0.53 & 12.13 & 145 & 75 & 72,65 \\
\hline 051229 & $01: 55$ & $\mathrm{ACE}$ & $0.18,-0.55,-0.82$ & $-0.16, \quad 0.54, \quad 0.83$ & 3.4 & 0.07 & 15.24 & 79 & 45 & $1, \quad 1$ \\
\hline \multirow[t]{3}{*}{051229} & $02: 28$ & $\mathrm{ACE}$ & & $0.64, \quad 0.78, \quad 0.15$ & 2.7 & -0.50 & 16.80 & & & 76,73 \\
\hline & $02: 28$ & $\mathrm{C} 1$ & $-0.47,-0.87,-0.14$ & $\begin{array}{lll}0.50, & 0.85, & 0.17\end{array}$ & 3.0 & 0.23 & 12.34 & 117 & 69 & \\
\hline & $02: 28$ & $\mathrm{C} 2$ & & $\begin{array}{lll}0.42, & 0.89, & 0.18\end{array}$ & 1.8 & 0.08 & 21.17 & & & \\
\hline 060117 & $04: 50$ & $\mathrm{C} 3$ & $-0.16,-0.75,-0.64$ & $-0.21, \quad 0.82, \quad 0.53$ & 1.4 & 0.99 & 26.55 & 99 & 107 & 57,53 \\
\hline \multirow[t]{3}{*}{060126} & $21: 22$ & $\mathrm{C} 1$ & & $0.38, \quad 0.92, \quad 0.06$ & 2.4 & -0.70 & 18.30 & & & 54,57 \\
\hline & $21: 22$ & $\mathrm{C} 2$ & $-0.72,-0.70, \quad 0.03$ & $\begin{array}{lll}0.28, & 0.96, & 0.07\end{array}$ & 4.4 & -1.07 & 11.74 & 135 & 154 & \\
\hline & $21: 22$ & $\mathrm{C} 3$ & & $\begin{array}{lll}0.47, & 0.88, & 0.03\end{array}$ & 4.1 & -0.52 & 12.70 & & & \\
\hline \multirow[t]{2}{*}{060128} & 05:56 & $\mathrm{C} 2$ & & $0.34,-0.17, \quad 0.92$ & 1.6 & 0.19 & 20.26 & & & 76,78 \\
\hline & $05: 56$ & $\mathrm{C} 3$ & $0.03,-0.14, \quad 0.99$ & $\begin{array}{lll}0.51, & 0.85, & 0.12\end{array}$ & 2.2 & 0.60 & 14.34 & 88 & 55 & \\
\hline \multirow[t]{2}{*}{060128} & $06: 12$ & $\mathrm{C} 1$ & & $0.39,-0.63, \quad 0.68$ & 1.4 & 0.07 & 27.53 & & & 72, 29 \\
\hline & $06: 12$ & $\mathrm{C} 4$ & $-0.23, \quad 0.73,-0.64$ & $0.29,-0.71, \quad 0.64$ & 1.9 & 0.02 & 19.34 & 103 & 113 & \\
\hline 060128 & $07: 24$ & $\mathrm{ACE}$ & $0.60,-0.78, \quad 0.17$ & $-0.40, \quad 0.91,-0.07$ & 1.5 & 0.74 & 32.51 & 53 & 30 & 34,35 \\
\hline \multirow[t]{4}{*}{060128} & $08: 25$ & $\mathrm{C} 1$ & & $-0.61,-0.19, \quad 0.77$ & 3.1 & -0.71 & 12.54 & & & 46,45 \\
\hline & $08: 25$ & $\mathrm{C} 2$ & & $-0.56,-0.18, \quad 0.81$ & 2.3 & -0.57 & 15.86 & & & \\
\hline & $08: 25$ & $\mathrm{C} 3$ & & $-0.58,-0.13, \quad 0.80$ & 3.1 & -0.76 & 12.58 & & & \\
\hline & $08: 25$ & $\mathrm{C} 4$ & $0.36, \quad 0.03,-0.93$ & $-0.59,-0.13, \quad 0.80$ & 4.0 & -0.72 & 10.54 & 68 & 65 & \\
\hline 060128 & $13: 25$ & $\mathrm{C} 2$ & $-0.21,-0.96, \quad 0.20$ & $0.28, \quad 0.90,-0.33$ & 1.30 & 0.08 & 36.73 & 102 & 51 & 43,45 \\
\hline \multirow[t]{2}{*}{060214} & $22: 35$ & $\mathrm{C} 3$ & & $-0.18,-0.57, \quad 0.80$ & 2.6 & -0.17 & 11.70 & & & 45,24 \\
\hline & $22: 35$ & $\mathrm{C} 4$ & $0.25, \quad 0.67,-0.70$ & $-0.27,-0.66, \quad 0.70$ & 7.3 & 0.08 & 5.95 & 75 & 43 & \\
\hline \multirow[t]{3}{*}{060215} & $23: 29$ & $\mathrm{C} 1$ & & $\begin{array}{lll}0.50, & 0.22, & 0.84\end{array}$ & 1.3 & -0.34 & 24.82 & & & $\mathbf{8 8}, 33$ \\
\hline & $23: 29$ & $\mathrm{C} 2$ & & $\begin{array}{lll}0.36, & 0.20, & 0.91\end{array}$ & 1.6 & -0.26 & 16.32 & & & \\
\hline & $23: 29$ & $\mathrm{C} 4$ & $\begin{array}{lll}0.37, & 0.25, & 0.89\end{array}$ & $\begin{array}{lll}0.07, & 0.15, & 0.99\end{array}$ & 4.0 & 1.00 & 9.63 & 68 & 130 & \\
\hline \multirow[t]{2}{*}{060221} & $01: 47$ & ACE & $-0.36,-0.26,-0.89$ & $\begin{array}{lll}0.22, & 0.36, & 0.91\end{array}$ & 5.90 & -0.54 & 8.89 & 111 & 89 & 34,30 \\
\hline & $01: 47$ & $\mathrm{C} 1$ & & $\begin{array}{lll}-0.16, & 0.31, & 0.93\end{array}$ & 1.4 & 0.48 & 26.59 & & & \\
\hline 060222 & 01:10 & $\mathrm{C} 3$ & $\begin{array}{lll}0.39, & 0.76, & 0.52\end{array}$ & $0.48, \quad 0.72, \quad 0.50$ & 2.30 & 0.24 & 23.05 & 66 & 107 & 80,84 \\
\hline \multirow[t]{2}{*}{060223} & $04: 14$ & $\mathrm{C} 2$ & & $0.62,-0.26,-0.74$ & 2.2 & -0.16 & 21.28 & & & \\
\hline & $04: 14$ & $\mathrm{C} 3$ & $0.62,-0.39,-0.68$ & $0.59,-0.41,-0.69$ & 2.3 & 0.12 & 20.69 & 51 & 7 & 50,50 \\
\hline \multirow[t]{2}{*}{060310} & $15: 30$ & $\mathrm{C} 3$ & $0.96,-0.26, \quad 0.14$ & $0.99,-0.05, \quad 0.12$ & 4.1 & -0.35 & 13.06 & 17 & 78 & 41,44 \\
\hline & $15: 30$ & $\mathrm{C} 4$ & & $0.94,-0.24, \quad 0.23$ & 3.9 & -0.27 & 13.39 & & & \\
\hline \multirow[t]{3}{*}{060320} & $04: 15$ & $\mathrm{C} 1$ & & $0.55,-0.21, \quad 0.81$ & 2.7 & 1.00 & 11.08 & & & 64,71 \\
\hline & $04: 15$ & $\mathrm{C} 2$ & $-0.19, \quad 0.17,-0.97$ & $0.36,-0.15, \quad 0.92$ & 5.2 & 0.67 & 8.18 & 101 & 90 & \\
\hline & $04: 15$ & $\mathrm{C} 4$ & & $0.68,-0.41, \quad 0.61$ & 2.0 & 1.47 & 15.12 & & & \\
\hline \multirow[t]{3}{*}{060322} & $07: 58$ & $\mathrm{C} 1$ & $\begin{array}{lll}0.56, & 0.43, & 0.71\end{array}$ & $0.75,-0.09, \quad 0.66$ & 5.2 & 0.66 & 9.38 & 56 & 125 & 16,27 \\
\hline & $07: 58$ & $\mathrm{C} 2$ & & $0.74,-0.10, \quad 0.67$ & 4.6 & 0.69 & 10.09 & & & \\
\hline & $07: 58$ & $\mathrm{C} 3$ & & $0.74,-0.23, \quad 0.63$ & 1.8 & 0.78 & 21.30 & & & \\
\hline 060410 & $04: 38$ & $\mathrm{C} 4$ & $\begin{array}{lll}0.69, & 0.14, & 0.71\end{array}$ & $\begin{array}{lll}0.54, & 0.23, & 0.81\end{array}$ & 1.3 & 0.98 & 29.94 & 46 & 42 & 67,66 \\
\hline
\end{tabular}


Table 2. Continued.

\begin{tabular}{|c|c|c|c|c|c|c|c|c|c|c|}
\hline $\begin{array}{c}\text { date } \\
\text { (yymmdd) }\end{array}$ & $\begin{array}{l}\text { time } \\
\text { (UT) }\end{array}$ & $\mathrm{s} / \mathrm{c}$ & $\boldsymbol{n}_{\boldsymbol{B}_{\mathrm{u}} \times \boldsymbol{B}_{\mathrm{d}}}$ & $\boldsymbol{n}_{\text {minvar }}$ & $\frac{\lambda_{2}}{\lambda_{3}}$ & $\begin{array}{l}B_{\min } \\
(n T)\end{array}$ & $\begin{array}{l}\Delta \boldsymbol{n} \\
\left({ }^{o}\right)\end{array}$ & $\begin{array}{c}\gamma \\
(o)\end{array}$ & $\begin{array}{c}\Delta \Phi \\
\left({ }^{o}\right)\end{array}$ & $\begin{array}{c}\theta_{C 1, C 3} \\
\left({ }^{o}\right)\end{array}$ \\
\hline \multirow[t]{3}{*}{060410} & $05: 28$ & $\mathrm{C} 1$ & & $\begin{array}{lll}0.66, & 0.39, & 0.64\end{array}$ & 1.4 & -0.96 & 35.69 & & & 41,43 \\
\hline & $05: 28$ & $\mathrm{C} 3$ & $\begin{array}{lll}0.53, & 0.56, & 0.64\end{array}$ & $\begin{array}{lll}0.49, & 0.59, & 0.64\end{array}$ & 1.7 & 0.15 & 27.49 & 58 & 116 & \\
\hline & $05: 28$ & $\mathrm{C} 4$ & & $\begin{array}{lll}0.46, & 0.57, & 0.68\end{array}$ & 1.4 & -0.55 & 39.17 & & & \\
\hline \multirow[t]{2}{*}{060410} & $07: 53$ & $\mathrm{C} 2$ & $\begin{array}{lll}0.60, & 0.18, & 0.78\end{array}$ & $\begin{array}{lll}0.76, & 0.16, & 0.63\end{array}$ & 2.3 & -0.83 & 15.52 & 53 & 42 & 32,32 \\
\hline & $07: 53$ & $\mathrm{C} 4$ & & $0.62,-0.08, \quad 0.78$ & 1.9 & -1.01 & 20.03 & & & \\
\hline \multirow[t]{4}{*}{060410} & 08:30 & $\mathrm{C} 1$ & & $0.84,-0.01, \quad 0.55$ & 1.1 & -1.03 & 43.56 & & & 30,34 \\
\hline & 08:30 & $\mathrm{C} 2$ & & $\begin{array}{lll}0.80, & 0.07, & 0.60\end{array}$ & 3.7 & -0.72 & 11.81 & & & \\
\hline & $08: 30$ & $\mathrm{C} 3$ & $\begin{array}{lll}0.62, & 0.39, & 0.68\end{array}$ & $\begin{array}{lll}0.76, & 0.13, & 0.64\end{array}$ & 4.9 & -0.62 & 9.79 & 51 & 114 & \\
\hline & $08: 30$ & $\mathrm{C} 4$ & & $0.76, \quad 0.11, \quad 0.64$ & 4.7 & -0.67 & 9.95 & & & \\
\hline \multirow[t]{2}{*}{060410} & $12: 52$ & $\mathrm{C} 1$ & $-0.15,-0.12, \quad 0.98$ & $\begin{array}{lll}-0.57, & 0.05, & 0.82\end{array}$ & 2.6 & 1.59 & 15.89 & 98 & 17 & 81,81 \\
\hline & $12: 52$ & $\mathrm{C} 2$ & & $0.84, \quad 0.18,-0.51$ & 1.7 & -0.85 & 24.58 & & & \\
\hline 060416 & $12: 38$ & $\mathrm{C} 2$ & $\begin{array}{lll}-0.37, & 0.23, & 0.90\end{array}$ & $\begin{array}{lll}0.49, & 0.75, & 0.43\end{array}$ & 1.3 & -0.07 & 28.76 & 112 & 8 & 39,60 \\
\hline \multirow[t]{2}{*}{060416} & $12: 45$ & $\mathrm{C} 1$ & $-0.06,-0.69,-0.72$ & $-0.09, \quad 0.77, \quad 0.63$ & 5.4 & -0.75 & 11.20 & 93 & 36 & \\
\hline & $12: 45$ & $\mathrm{C} 2$ & & $\begin{array}{lll}0.09, & 0.73, & 0.68\end{array}$ & 2.1 & 0.46 & 22.20 & & & \\
\hline 060416 & $13: 24$ & $\mathrm{C} 1$ & $\begin{array}{lll}0.77, & 0.54, & 0.33\end{array}$ & $\begin{array}{lll}0.73, & 0.36, & 0.58\end{array}$ & 1.5 & 0.30 & 35.29 & 39 & 36 & \\
\hline 060416 & $15: 56$ & $\mathrm{C} 4$ & $0.23,-0.59, \quad 0.77$ & $0.44,-0.26, \quad 0.86$ & 6.4 & -0.38 & 7.77 & 76 & 25 & 48,47 \\
\hline \multirow[t]{2}{*}{060416} & $16: 29$ & $\mathrm{C} 1$ & & $\begin{array}{lll}0.31, & 0.63, & 0.71\end{array}$ & 6.0 & 0.52 & 9.24 & & & 43,54 \\
\hline & $16: 29$ & $\mathrm{C} 2$ & $0.01,-0.81,-0.58$ & $\begin{array}{lll}0.14, & 0.75, & 0.65\end{array}$ & 14.4 & 0.30 & 5.63 & 89 & 126 & \\
\hline \multirow[t]{2}{*}{060416} & $16: 40$ & $\mathrm{ACE}$ & $-0.78,-0.37,-0.51$ & $\begin{array}{lll}0.85, & 0.12, & 0.52\end{array}$ & 1.7 & 0.44 & 20.40 & 140 & 109 & 43,54 \\
\hline & $16: 40$ & $\mathrm{C} 1$ & & $0.83,-0.08,-0.55$ & 1.4 & -0.11 & 29.50 & & & \\
\hline 060416 & $18: 33$ & $\mathrm{ACE}$ & $0.30,-0.09,-0.95$ & $-0.48,-0.17, \quad 0.86$ & 3.5 & -0.35 & 12.03 & 72 & 22 & 44,49 \\
\hline \multirow[t]{2}{*}{060416} & 20:01 & C3 & $-0.05, \quad 0.29, \quad 0.96$ & $\begin{array}{lll}0.17, & 0.44, & 0.88\end{array}$ & 2.0 & -0.03 & 14.97 & 92 & 10 & 49,48 \\
\hline & 20:01 & $\mathrm{C} 4$ & & $\begin{array}{lll}0.29, & 0.43, & 0.85\end{array}$ & 1.5 & -0.20 & 21.62 & & & \\
\hline 070104 & $03: 54$ & $\mathrm{C} 2$ & $0.83,-0.33, \quad 0.45$ & $\begin{array}{lll}0.25, & 0.93, & 0.29\end{array}$ & 1.3 & -0.50 & 32.78 & 33 & 27 & 44,44 \\
\hline 070104 & $04: 38$ & $\mathrm{ACE}$ & $-0.63, \quad 0.64,-0.45$ & $0.68,-0.72, \quad 0.13$ & 10.3 & -1.48 & 7.73 & 128 & 26 & 31,31 \\
\hline \multirow[t]{5}{*}{070104} & 05:08 & $\mathrm{ACE}$ & & $0.60,-0.54, \quad 0.58$ & 2.2 & -0.03 & 19.08 & & & \\
\hline & 05:08 & $\mathrm{C} 1$ & & $0.71,-0.61, \quad 0.35$ & 2.9 & -0.16 & 14.68 & & & \\
\hline & 05:08 & $\mathrm{C} 2$ & & $0.57,-0.68, \quad 0.46$ & 2.1 & 0.12 & 19.78 & & & \\
\hline & 05:08 & $\mathrm{C} 3$ & $0.64,-0.64, \quad 0.43$ & $0.68,-0.62, \quad 0.40$ & 3.0 & -0.15 & 13.51 & 50 & 113 & \\
\hline & 05:08 & $\mathrm{C} 4$ & & $0.58,-0.67, \quad 0.46$ & 2.6 & 0.10 & 16.49 & & & \\
\hline \multirow[t]{2}{*}{070104} & $06: 20$ & ACE & $\begin{array}{lll}0.62, & 0.04, & 0.78\end{array}$ & $\begin{array}{lll}0.61, & 0.05, & 0.79\end{array}$ & 7.1 & 0.04 & 8.63 & 51 & 96 & 23,23 \\
\hline & 06:20 & $\mathrm{C} 1$ & & $\begin{array}{lll}0.73, & 0.30, & 0.62\end{array}$ & 1.8 & -0.05 & 20.18 & & & \\
\hline 070106 & $16: 10$ & $\mathrm{C} 1$ & $\begin{array}{lll}0.24, & 0.44, & 0.86\end{array}$ & $0.21, \quad 0.76, \quad 0.61$ & 1.7 & 0.66 & 20.21 & 75 & 8 & $10, \quad 5$ \\
\hline \multirow[t]{5}{*}{070108} & $11: 25$ & $\mathrm{ACE}$ & & $-0.51, \quad 0.86,-0.01$ & 2.2 & 0.03 & 17.13 & & & 82,74 \\
\hline & $11: 25$ & $\mathrm{C} 1$ & $-0.62, \quad 0.78, \quad 0.01$ & $\begin{array}{lll}-0.66, & 0.75, & 0.02\end{array}$ & 2.4 & -0.04 & 12.24 & 128 & 129 & \\
\hline & $11: 25$ & $\mathrm{C} 2$ & & $-0.67, \quad 0.74, \quad 0.06$ & 2.1 & -0.05 & 15.71 & & & \\
\hline & $11: 25$ & $\mathrm{C} 3$ & & $-0.50, \quad 0.86,-0.06$ & 1.2 & 0.04 & 37.42 & & & \\
\hline & $11: 25$ & $\mathrm{C} 4$ & & $0.50,-0.86, \quad 0.05$ & 1.3 & -0.08 & 30.75 & & & \\
\hline 070116 & 09:41 & $\mathrm{C} 1$ & $\begin{array}{lll}0.36, & 0.29, & 0.88\end{array}$ & $\begin{array}{lll}0.36, & 0.17, & 0.92\end{array}$ & 2.3 & -0.37 & 14.57 & 68 & 6 & 24,22 \\
\hline 070116 & $10: 00$ & $\mathrm{ACE}$ & $0.98,-0.11, \quad 0.15$ & $0.94,-0.29,-0.17$ & 2.2 & 0.25 & 17.96 & 10 & 160 & 21,16 \\
\hline \multirow[t]{3}{*}{070116} & $10: 50$ & $\mathrm{C} 1$ & & $0.74,-0.64, \quad 0.20$ & 3.9 & -0.12 & 9.57 & & & 28,28 \\
\hline & $10: 50$ & $\mathrm{C} 2$ & & $0.73,-0.63, \quad 0.26$ & 3.0 & -0.11 & 11.49 & & & \\
\hline & $10: 50$ & $\mathrm{C} 3$ & $-0.73, \quad 0.61,-0.31$ & $0.68,-0.61, \quad 0.40$ & 4.4 & 0.22 & 8.72 & 136 & 43 & \\
\hline 070116 & $10: 50$ & $\mathrm{C} 4$ & & $0.69,-0.62, \quad 0.37$ & 4.4 & 0.16 & 8.70 & & & \\
\hline 070117 & $16: 40$ & $\mathrm{ACE}$ & $-0.49,-0.74,-0.46$ & $\begin{array}{lll}0.55, & 0.55, & 0.63\end{array}$ & 1.9 & -0.80 & 24.59 & 119 & 82 & 9,12 \\
\hline 070118 & $07: 52$ & $\mathrm{C} 3$ & $\begin{array}{lll}0.50, & 0.85, & 0.14\end{array}$ & $\begin{array}{lll}0.45, & 0.88, & 0.18\end{array}$ & 1.5 & 0.30 & 25.86 & 59 & 57 & 28,24 \\
\hline 070118 & $07: 52$ & $\mathrm{C} 4$ & & $\begin{array}{lll}0.51, & 0.85, & 0.12\end{array}$ & 1.1 & -0.19 & 48.52 & & & \\
\hline 070118 & $09: 38$ & $\mathrm{C} 2$ & $0.85,-0.25, \quad 0.46$ & $0.87,-0.29, \quad 0.39$ & 1.5 & -0.33 & 22.97 & 31 & 55 & 26,17 \\
\hline 070118 & 09:44 & $\mathrm{C} 1$ & $\begin{array}{lll}0.76, & 0.27, & 0.58\end{array}$ & $\begin{array}{lll}0.76, & 0.24, & 0.60\end{array}$ & 2.3 & 0.04 & 15.52 & 40 & 137 & \\
\hline
\end{tabular}


Table 2. Continued.

\begin{tabular}{|c|c|c|c|c|c|c|c|c|c|c|}
\hline $\begin{array}{c}\text { date } \\
\text { (yymmdd) }\end{array}$ & $\begin{array}{l}\text { time } \\
\text { (UT) }\end{array}$ & $\mathrm{s} / \mathrm{c}$ & $\boldsymbol{n}_{\boldsymbol{B}_{\mathrm{u}} \times \boldsymbol{B}_{\mathrm{d}}}$ & $\boldsymbol{n}_{\text {minvar }}$ & $\frac{\lambda_{2}}{\lambda_{3}}$ & $\begin{array}{l}B_{\min } \\
(n T)\end{array}$ & $\begin{array}{l}\Delta \boldsymbol{n} \\
\left({ }^{o}\right)\end{array}$ & $\begin{array}{c}\gamma \\
(o)\end{array}$ & $\begin{array}{c}\Delta \Phi \\
\left({ }^{o}\right)\end{array}$ & $\begin{array}{c}\theta_{C 1, C 3} \\
\left({ }^{o}\right)\end{array}$ \\
\hline 070118 & $12: 15$ & $\mathrm{C} 1$ & $-0.80,-0.10, \quad 0.59$ & $-0.84, \quad 0.08,-0.53$ & 2.9 & -0.18 & 12.16 & 143 & 87 & $38, \quad 9$ \\
\hline \multirow[t]{3}{*}{070118} & $14: 36$ & $\mathrm{C} 1$ & & $0.07, \quad 1.00,-0.04$ & 2.2 & 0.03 & 13.11 & & & 36,14 \\
\hline & $14: 36$ & $\mathrm{C} 2$ & & $0.04, \quad 1.00,-0.02$ & 2.7 & 0.05 & 10.83 & & & \\
\hline & $14: 36$ & $\mathrm{C} 3$ & $-0.02,-1.00, \quad 0.02$ & $0.03, \quad 1.00,-0.15$ & 2.9 & -0.60 & 10.69 & 91 & 28 & \\
\hline \multirow[t]{4}{*}{070118} & $19: 35$ & $\mathrm{C} 1$ & & $0.52, \quad 0.79,-0.33$ & 2.7 & -0.18 & 12.26 & & & 87,87 \\
\hline & $19: 35$ & $\mathrm{C} 2$ & & $0.55, \quad 0.77,-0.31$ & 2.1 & 0.01 & 14.83 & & & \\
\hline & $19: 35$ & $\mathrm{C} 3$ & & $0.41, \quad 0.80,-0.44$ & 2.4 & 0.23 & 13.41 & & & \\
\hline & $19: 35$ & $\mathrm{C} 4$ & $0.50, \quad 0.77,-0.39$ & $0.46, \quad 0.80,-0.40$ & 3.3 & 0.10 & 10.51 & 60 & 81 & \\
\hline \multirow[t]{4}{*}{070120} & $18: 20$ & $\mathrm{C} 1$ & & $0.69,-0.40, \quad 0.60$ & 7.2 & -0.42 & 6.28 & & & \\
\hline & $18: 20$ & $\mathrm{C} 2$ & $0.51,-0.42, \quad 0.75$ & $0.60,-0.41, \quad 0.69$ & 10.3 & -0.31 & 5.19 & 59 & 100 & \\
\hline & $18: 20$ & $\mathrm{C} 3$ & & $0.61,-0.40, \quad 0.68$ & 8.3 & -0.40 & 5.89 & & & \\
\hline & $18: 20$ & $\mathrm{C} 4$ & & $0.63,-0.41, \quad 0.66$ & 7.6 & -0.51 & 6.14 & & & \\
\hline 070130 & $16: 47$ & $\mathrm{ACE}$ & $0.67,-0.58, \quad 0.46$ & $0.83,-0.46, \quad 0.31$ & 1.8 & -0.80 & 28.51 & 47 & 26 & 23,17 \\
\hline \multirow[t]{4}{*}{070201} & $06: 49$ & $\mathrm{C} 1$ & $0.24,-0.33, \quad 0.91$ & $0.26,-0.33, \quad 0.91$ & 2.2 & -0.07 & 15.73 & 75 & 71 & 41,44 \\
\hline & $06: 49$ & $\mathrm{C} 2$ & & $-0.11,-0.39, \quad 0.91$ & 1.0 & 0.57 & 104.85 & & & \\
\hline & $06: 49$ & $\mathrm{C} 3$ & & $-0.20,-0.47, \quad 0.86$ & 1.3 & 0.87 & 32.17 & & & \\
\hline & $06: 49$ & $\mathrm{C} 4$ & & $-0.11,-0.47, \quad 0.88$ & 1.2 & 0.66 & 41.45 & & & \\
\hline \multirow[t]{4}{*}{070201} & $22: 08$ & $\mathrm{C} 1$ & $0.30,-0.89, \quad 0.33$ & $-0.35, \quad 0.88,-0.31$ & 3.0 & 0.05 & 12.58 & 72 & 37 & 64,61 \\
\hline & 22:08 & $\mathrm{C} 2$ & & $-0.38, \quad 0.90,-0.21$ & 2.6 & 0.65 & 13.92 & & & \\
\hline & 22:08 & $\mathrm{C} 3$ & & $-0.43, \quad 0.86,-0.29$ & 2.7 & 0.16 & 13.66 & & & \\
\hline & 22:08 & $\mathrm{C} 4$ & & $-0.42, \quad 0.85,-0.30$ & 1.9 & 0.16 & 18.62 & & & \\
\hline \multirow[t]{2}{*}{070201} & $22: 17$ & ACE & $0.22,-0.89, \quad 0.41$ & $0.15,-0.89, \quad 0.44$ & 16.9 & 0.16 & 6.57 & 77 & 64 & $\mathbf{5 7}, 43$ \\
\hline & $22: 17$ & $\mathrm{C} 3$ & & $-0.23, \quad 0.84,-0.48$ & 2.1 & 0.13 & 21.55 & & & \\
\hline \multirow[t]{3}{*}{070202} & $01: 31$ & $\mathrm{ACE}$ & & $-0.42, \quad 0.91, \quad 0.02$ & 2.5 & 0.08 & 15.51 & & & 83,83 \\
\hline & $01: 31$ & $\mathrm{C} 1$ & $0.53,-0.84,-0.10$ & $-0.57, \quad 0.80, \quad 0.18$ & 4.0 & 0.29 & 9.43 & 58 & 25 & \\
\hline & $01: 31$ & $\mathrm{C} 2$ & & $-0.44, \quad 0.89, \quad 0.05$ & 3.0 & -0.26 & 12.34 & & & \\
\hline \multirow[t]{2}{*}{070209} & $02: 16$ & $\mathrm{C} 1$ & $-0.96,-0.25, \quad 0.14$ & $0.94, \quad 0.29,-0.16$ & 5.8 & -0.13 & 8.62 & 163 & 89 & 72,63 \\
\hline & $02: 16$ & $\mathrm{C} 2$ & & $0.92, \quad 0.35,-0.19$ & 5.8 & -0.16 & 8.68 & & & \\
\hline 070215 & 01:35 & $\mathrm{C} 2$ & $0.31,-0.05,-0.95$ & $-0.38, \quad 0.14, \quad 0.91$ & 2.2 & 0.31 & 14.35 & 71 & 55 & 25,28 \\
\hline \multirow[t]{4}{*}{070215} & $02: 31$ & $\mathrm{ACE}$ & & $0.66,-0.21, \quad 0.73$ & 3.0 & 0.01 & 13.32 & & & 25,28 \\
\hline & $02: 31$ & $\mathrm{C} 1$ & $0.72,-0.33, \quad 0.61$ & $0.70,-0.33, \quad 0.63$ & 5.9 & 0.06 & 6.73 & 43 & 102 & \\
\hline & $02: 31$ & $\mathrm{C} 3$ & & $0.55,-0.35, \quad 0.75$ & 1.6 & -0.05 & 19.96 & & & \\
\hline & $02: 31$ & $\mathrm{C} 4$ & & $0.52,-0.37, \quad 0.77$ & 1.7 & 0.10 & 15.51 & & & \\
\hline \multirow[t]{5}{*}{070215} & $02: 50$ & $\mathrm{ACE}$ & & $-0.40,-0.22, \quad 0.89$ & 3.7 & -0.11 & 13.76 & & & 25,28 \\
\hline & $02: 50$ & $\mathrm{C} 1$ & & $0.63,-0.06, \quad 0.77$ & 1.2 & -0.14 & 26.49 & & & \\
\hline & $02: 50$ & $\mathrm{C} 2$ & $0.14, \quad 0.19,-0.97$ & $0.80, \quad 0.30,-0.53$ & 4.5 & -1.09 & 5.53 & 82 & 138 & \\
\hline & $02: 50$ & $\mathrm{C} 3$ & & $0.66,-0.17, \quad 0.73$ & 1.6 & 0.00 & 16.97 & & & \\
\hline & $02: 50$ & $\mathrm{C} 4$ & & $0.68,-0.17, \quad 0.71$ & 1.4 & 0.00 & 19.53 & & & \\
\hline 070215 & $03: 13$ & $\mathrm{C} 1$ & $0.43,-0.27,-0.86$ & $0.70,-0.54,-0.47$ & 1.6 & -0.27 & 24.17 & 64 & 8 & 27,29 \\
\hline 070215 & $03: 56$ & $\mathrm{C} 4$ & $0.43, \quad 0.31, \quad 0.85$ & $\begin{array}{lll}0.66, & 0.35, & 0.66\end{array}$ & 9.1 & -1.25 & 6.78 & 64 & 100 & 25,25 \\
\hline 070215 & 04:00 & $\mathrm{C} 1$ & $-0.06,-0.71,-0.70$ & $\begin{array}{lll}0.05, & 0.75, & 0.65\end{array}$ & 1.9 & -0.13 & 20.13 & 93 & 20 & \\
\hline \multirow[t]{4}{*}{070215} & $08: 45$ & $\mathrm{C} 1$ & $\begin{array}{lll}0.82, & 0.41, & 0.40\end{array}$ & $\begin{array}{lll}0.79, & 0.44, & 0.42\end{array}$ & 6.5 & -0.08 & 6.81 & 35 & 66 & 10,25 \\
\hline & $08: 45$ & $\mathrm{C} 2$ & & $\begin{array}{lll}0.84, & 0.17, & 0.51\end{array}$ & 2.3 & 0.10 & 16.65 & & & \\
\hline & $08: 45$ & $\mathrm{C} 3$ & & $\begin{array}{lll}0.75, & 0.26, & 0.61\end{array}$ & 1.6 & 0.18 & 26.22 & & & \\
\hline & $08: 45$ & $\mathrm{C} 4$ & & $\begin{array}{lll}0.70, & 0.20, & 0.68\end{array}$ & 1.6 & 0.29 & 25.26 & & & \\
\hline 070215 & $15: 15$ & $\mathrm{C} 3$ & $0.45,-0.53, \quad 0.72$ & $0.49,-0.53, \quad 0.69$ & 3.4 & -0.14 & 11.26 & 63 & 74 & $44, \mathbf{5 3}$ \\
\hline 070215 & $15: 15$ & $\mathrm{C} 4$ & & $0.48,-0.51, \quad 0.71$ & 2.7 & -0.08 & 13.36 & & & \\
\hline 070215 & $22: 08$ & $\mathrm{C} 2$ & & $-0.38, \quad 0.90,-0.21$ & 2.6 & 0.65 & 13.92 & & & \\
\hline 070301 & $04: 56$ & $\mathrm{C} 2$ & $0.75,-0.66,-0.12$ & $0.74,-0.67,-0.02$ & 1.9 & -0.10 & 15.83 & 41 & 62 & 35,35 \\
\hline
\end{tabular}


Table 2. Continued.

\begin{tabular}{|c|c|c|c|c|c|c|c|c|c|c|}
\hline $\begin{array}{c}\text { date } \\
\text { (yymmdd) }\end{array}$ & $\begin{array}{l}\text { time } \\
\text { (UT) }\end{array}$ & $\mathrm{s} / \mathrm{c}$ & $\boldsymbol{n}_{\boldsymbol{B}_{\mathrm{u}} \times \boldsymbol{B}_{\mathrm{d}}}$ & $\boldsymbol{n}_{\text {minvar }}$ & $\frac{\lambda_{2}}{\lambda_{3}}$ & $\begin{array}{l}B_{\min } \\
(n T)\end{array}$ & $\begin{array}{l}\Delta \boldsymbol{n} \\
\left({ }^{o}\right)\end{array}$ & $\begin{array}{c}\gamma \\
(o)\end{array}$ & $\begin{array}{c}\Delta \Phi \\
(o)\end{array}$ & $\begin{array}{c}\theta_{C 1, C 3} \\
\left({ }^{o}\right)\end{array}$ \\
\hline \multirow[t]{2}{*}{070301} & $04: 56$ & $\mathrm{C} 3$ & & $0.64,-0.77,-0.06$ & 1.2 & -0.05 & 32.86 & & & \\
\hline & $04: 56$ & $\mathrm{C} 4$ & & $0.64,-0.76,-0.11$ & 1.2 & 0.07 & 35.48 & & & \\
\hline \multirow[t]{3}{*}{070301} & $07: 11$ & $\mathrm{C} 1$ & & $0.97,-0.13, \quad 0.20$ & 2.2 & -0.15 & 12.07 & & & 20,21 \\
\hline & $07: 11$ & $\mathrm{C} 2$ & $-0.93, \quad 0.16,-0.33$ & $0.93,-0.15, \quad 0.35$ & 2.9 & 0.03 & 9.77 & 158 & 99 & \\
\hline & $07: 11$ & $\mathrm{C} 3$ & & $0.92,-0.18, \quad 0.34$ & 2.5 & -0.08 & 10.93 & & & \\
\hline \multirow[t]{2}{*}{070301} & $09: 43$ & $\mathrm{C} 1$ & & $0.47,-0.80,-0.37$ & 1.7 & 0.21 & 15.12 & & & 87,89 \\
\hline & $09: 43$ & $\mathrm{C} 2$ & $0.72,-0.69,-0.03$ & $0.78,-0.60,-0.15$ & 2.0 & -0.34 & 12.93 & 43 & 17 & \\
\hline 070301 & $10: 30$ & $\mathrm{C} 1$ & $-0.61, \quad 0.64,-0.46$ & $-0.56, \quad 0.79,-0.26$ & 1.9 & -0.29 & 15.42 & 127 & 8 & 16,23 \\
\hline 070302 & 02:03 & $\mathrm{C} 1$ & $\begin{array}{lll}0.71, & 0.69, & 0.12\end{array}$ & $\begin{array}{lll}0.80, & 0.57, & 0.18\end{array}$ & 2.1 & -0.17 & 12.71 & 44 & 8 & 47, 51 \\
\hline \multirow[t]{3}{*}{070313} & $05: 37$ & $\mathrm{C} 2$ & $0.60, \quad 0.52,-0.61$ & $0.61, \quad 0.52,-0.60$ & 5.2 & -0.05 & 7.48 & 53 & 92 & 24,23 \\
\hline & $05: 37$ & $\mathrm{C} 3$ & & $0.67, \quad 0.52,-0.53$ & 3.2 & -0.53 & 11.53 & & & \\
\hline & $05: 37$ & $\mathrm{C} 4$ & & $0.69, \quad 0.49,-0.52$ & 3.8 & -0.48 & 10.35 & & & \\
\hline 070314 & $07: 54$ & ACE & $\begin{array}{lll}0.37, & 0.32, & 0.87\end{array}$ & $\begin{array}{lll}0.38, & 0.29, & 0.88\end{array}$ & 2.9 & -0.14 & 15.87 & 68 & 68 & 39,39 \\
\hline \multirow[t]{3}{*}{070314} & $08: 37$ & $\mathrm{C} 1$ & & $\begin{array}{lll}0.74, & 0.43, & 0.52\end{array}$ & 1.8 & 0.27 & 30.34 & & & 45,39 \\
\hline & $08: 37$ & $\mathrm{C} 3$ & $\begin{array}{lll}0.76, & 0.31, & 0.57\end{array}$ & $\begin{array}{lll}0.77, & 0.00, & 0.64\end{array}$ & 4.0 & 0.08 & 15.22 & 40 & 17 & \\
\hline & $08: 37$ & $\mathrm{C} 4$ & & $\begin{array}{lll}0.70, & 0.20, & 0.68\end{array}$ & 2.9 & 0.28 & 18.32 & & & \\
\hline 070314 & $12: 51$ & ACE & $-0.17, \quad 0.21,-0.96$ & $0.30,-0.50, \quad 0.81$ & 2.0 & -0.18 & 22.90 & 99 & 26 & 51,51 \\
\hline 070314 & $15: 52$ & $\mathrm{ACE}$ & $-0.85,-0.46,-0.26$ & $0.86, \quad 0.52,-0.03$ & 1.9 & 0.16 & 21.58 & 147 & 163 & $9, \quad 9$ \\
\hline \multirow[t]{2}{*}{070315} & $12: 15$ & ACE & $0.40,-0.23, \quad 0.89$ & $0.48,-0.08, \quad 0.87$ & 2.2 & 0.32 & 20.23 & 66 & 109 & 58,58 \\
\hline & $12: 15$ & $\mathrm{C} 1$ & & $0.37,-0.40, \quad 0.84$ & 1.5 & -0.07 & 22.67 & & & \\
\hline 070316 & $18: 14$ & $\mathrm{C} 1$ & $-0.15,-0.98, \quad 0.12$ & $0.17, \quad 0.97,-0.18$ & 2.0 & -0.22 & 14.41 & 98 & 12 & 40,39 \\
\hline \multirow[t]{4}{*}{070316} & $19: 57$ & $\mathrm{C} 1$ & & $0.64,-0.61, \quad 0.46$ & 1.6 & -0.08 & 17.19 & & & 51,53 \\
\hline & $19: 57$ & $\mathrm{C} 2$ & & $0.56,-0.53, \quad 0.63$ & 1.6 & -0.02 & 16.57 & & & \\
\hline & $19: 57$ & $\mathrm{C} 3$ & & $0.41,-0.51, \quad 0.75$ & 1.2 & 0.13 & 30.65 & & & \\
\hline & $19: 57$ & $\mathrm{C} 4$ & $0.37,-0.39, \quad 0.84$ & $0.31,-0.50, \quad 0.81$ & 1.7 & 0.17 & 16.15 & 68 & 38 & \\
\hline 070319 & $03: 47$ & $\mathrm{C} 2$ & & $0.83, \quad 0.03, \quad 0.56$ & 1.1 & -0.04 & 48.73 & & & 49, 30 \\
\hline 070319 & $03: 47$ & $\mathrm{C} 4$ & $\begin{array}{lll}0.56, & 0.14, & 0.82\end{array}$ & $0.20, \quad 0.19, \quad 0.96$ & 2.1 & 0.30 & 14.07 & 56 & 144 & \\
\hline 070319 & $04: 28$ & $\mathrm{C} 1$ & & $\begin{array}{lll}0.50, & 0.42, & 0.76\end{array}$ & 2.1 & -0.14 & 13.22 & & & 16,13 \\
\hline 070319 & $04: 28$ & $\mathrm{C} 2$ & & $0.32, \quad 0.40, \quad 0.86$ & 1.9 & 0.02 & 15.32 & & & \\
\hline 070319 & $04: 28$ & $\mathrm{C} 3$ & $-0.24,-0.39,-0.89$ & $\begin{array}{lll}0.20, & 0.38, & 0.90\end{array}$ & 2.3 & 0.06 & 12.42 & 104 & 96 & \\
\hline \multirow[t]{2}{*}{070328} & $13: 41$ & $\mathrm{C} 3$ & $\begin{array}{lll}0.90, & 0.31, & 0.29\end{array}$ & $\begin{array}{lll}0.90, & 0.30, & 0.30\end{array}$ & 1.5 & -0.01 & 20.23 & 25 & 47 & $9, \mathbf{8 8}$ \\
\hline & $13: 41$ & $\mathrm{C} 4$ & & $\begin{array}{lll}0.86, & 0.38, & 0.34\end{array}$ & 1.5 & 0.11 & 20.58 & & & \\
\hline 070328 & $15: 22$ & $\mathrm{C} 2$ & $0.23,-0.93,-0.28$ & $-0.24, \quad 0.96,-0.15$ & 1.9 & -0.06 & 24.12 & 76 & 4 & 35,30 \\
\hline 070328 & $16: 08$ & $\mathrm{C} 2$ & $0.04, \quad 1.00, \quad 0.05$ & $\begin{array}{lll}-0.09, & 0.95, & 0.29\end{array}$ & 1.4 & -0.30 & 23.79 & 87 & 13 & 36,47 \\
\hline \multirow{3}{*}{070328} & $16: 51$ & $\mathrm{C} 1$ & & $0.63,-0.22,-0.74$ & 1.7 & 0.01 & 16.24 & & & 66, 71 \\
\hline & $16: 51$ & $\mathrm{C} 2$ & & $0.51,-0.24,-0.82$ & 2.0 & 0.00 & 13.11 & & & \\
\hline & $16: 51$ & $\mathrm{C} 4$ & $0.05,-0.15,-0.99$ & $\begin{array}{lll}-0.50, & 0.28, & 0.82\end{array}$ & 2.4 & -0.34 & 11.23 & 86 & 16 & \\
\hline 070429 & $20: 41$ & $\mathrm{C} 2$ & $-0.79,-0.55, \quad 0.29$ & $0.79, \quad 0.54,-0.29$ & 2.6 & 0.04 & 12.24 & 141 & 68 & 39,40 \\
\hline 070429 & $21: 00$ & $\mathrm{C} 1$ & $-0.79,-0.48, \quad 0.37$ & $0.72, \quad 0.35,-0.60$ & 1.4 & 0.66 & 18.29 & 142 & 27 & 51,52 \\
\hline 070429 & $22: 06$ & $\mathrm{C} 1$ & $-0.37,-0.90,-0.23$ & $\begin{array}{lll}0.33, & 0.85, & 0.41\end{array}$ & 1.2 & -0.52 & 27.17 & 111 & 44 & 39,40 \\
\hline \multirow[t]{5}{*}{070429} & 23:02 & ACE & $-0.77, \quad 0.14,-0.63$ & $0.72,-0.11, \quad 0.69$ & 4.9 & -0.18 & 10.03 & 140 & 115 & \\
\hline & 23:02 & $\mathrm{C} 1$ & & $0.66,-0.04, \quad 0.75$ & 1.8 & 0.14 & 14.56 & & & \\
\hline & $23: 02$ & $\mathrm{C} 2$ & & $0.72,-0.07, \quad 0.69$ & 1.9 & 0.26 & 13.85 & & & \\
\hline & 23:02 & $\mathrm{C} 3$ & & $0.65,-0.03, \quad 0.76$ & 2.3 & -0.09 & 11.31 & & & \\
\hline & $23: 02$ & $\mathrm{C} 4$ & & $0.71,-0.03, \quad 0.70$ & 1.9 & -0.04 & 15.05 & & & \\
\hline \multirow[t]{4}{*}{070430} & 02:02 & $\mathrm{C} 1$ & $-0.23,-0.46,-0.86$ & $\begin{array}{lll}0.65, & 0.40, & 0.64\end{array}$ & 6.3 & 0.65 & 5.92 & 103 & 146 & \\
\hline & 02:02 & $\mathrm{C} 2$ & & $\begin{array}{lll}0.67, & 0.40, & 0.62\end{array}$ & 5.6 & 0.70 & 6.39 & & & \\
\hline & 02:02 & $\mathrm{C} 3$ & & $\begin{array}{lll}0.68, & 0.40, & 0.61\end{array}$ & 5.5 & 0.48 & 6.50 & & & \\
\hline & 02:02 & $\mathrm{C} 4$ & & $\begin{array}{lll}0.67, & 0.41, & 0.62\end{array}$ & 5.0 & 0.26 & 6.86 & & & \\
\hline
\end{tabular}




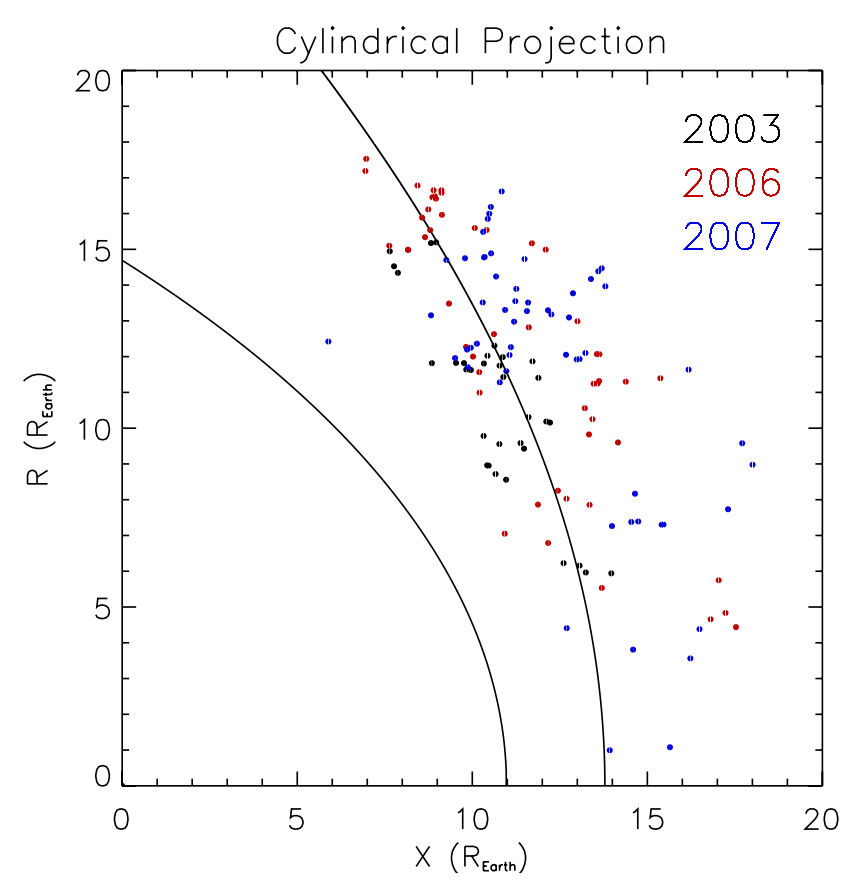

Fig. 2. Cylindrical projection of Cluster SC center positions during HFA observation and the average bow shock and magnetopause positions in GSE system. The shape of the magnetopause and the bow shock were calculated using the average solar wind pressure (Sibeck et al., 1991; Tsyganenko, 1995; Peredo et al., 1995). The black, red and blue points show the Cluster SC positions when HFA was observed in 2003, 2006 and 2007, respectively. The coordinates were plotted in $R_{\text {Earth }}$ units.

minimum variance method if the cross product method did not differ by more than $15^{\circ}$ and the ratio of second and third eigenvalues were equal to or larger than 2.0 (Table 2) (for a more detailed description of the method see Facskó et al., 2008; Facskó et al., 2009). It turns out that the minimum variance method can mostly be used at low magnetic field variation. This method is very difficult and almost impossible to use in the HFA cavity and in SLAMS (Short Large Amplitude Magnetic Structures) mostly coupled to quasiparallel regions (Schwartz and Burgess, 1991). Many HFAs were embedded into SLAMS and so we were able to use the minimum variance method with good accuracy only in a few cases. Beside of this feature of the method we have found more HFAs at the quasi-parallel region $(\sim 66 \%)$ (see Table 2$)$. The local bow shock normals were calculated by scaling a model bow shock to the spacecraft location as in Schwartz et al. (2000) and we used the upstream magnetic field upstream of the HFA to calculate the angle of the shock-normal and the magnetic field vector. This might confirm previous results: the conditions were quasi-parallel at least on one side of the TD previously (see Onsager et al., 1991; Thomsen et al., 1993; Kecskeméty et al., 2006) and current simulations expect the HFAs to appear where the quasi-parallel condition turns to quasi-perpendicular (Omidi and Sibeck, 2007). We

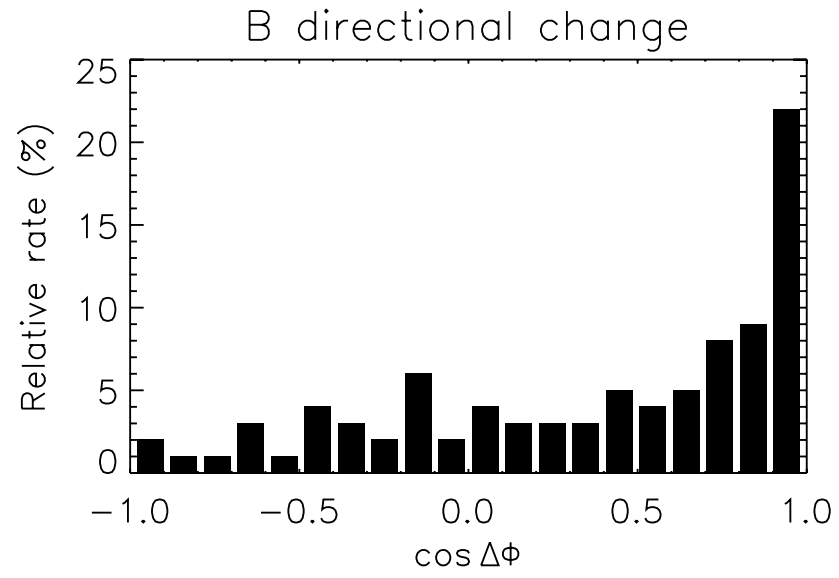

Fig. 3. Distribution of $\cos \Delta \Phi$ where $\Delta \Phi$ is the angle of magnetic field directional change at the discontinuity.

used the same conditions for HFA observation and determination in 2003 (Kecskeméty et al., 2006; Facskó et al., 2008), 2006 and 2007 (Facskó et al., 2009), however this effect was very strong in 2007 and it was also noticeable in 2003 and 2006.

The $\Delta \Phi$ and $\gamma$ distributions differ from the typical distributions associated with discontinuities in the solar wind. The $\Delta \Phi$ distribution associated with HFAs (Fig. 3) peaks at smaller values $\left(0^{\circ}-30^{\circ}\right)$ when compared to the distribution of solar wind distribution rotation angles, which peaks at larger values $\left(30^{\circ}-45^{\circ}\right.$, Knetter et al., 2004, Fig. 2). The $\gamma$ distribution associated with HFAs (Fig. 4) shows a wide, empty cone around the Sun-Earth line, which is in contrast to the distributor of solar wind discontinuities, whose normals typically have small $\gamma$ angles (Knetter et al., 2004, Fig. 11). We found only one normal vector within this cone in 2003 and a few others in 2006 and 2007. We observed this feature in the distribution of $\gamma$ (Fig. 4). This finding strongly supports the earlier theoretical and simulation results that HFAs can only be formed if $43^{\circ} \leq \gamma \leq 83^{\circ}$ (Lin, 2002; Németh, 2007; Facskó et al., 2008). The distribution of $\Delta \Phi$ shows that HFAs can be formed if the magnetic field vector directional change is sufficiently large across the TD (Table 2). Actually smaller values of $\Delta \Phi$ were also observed, which supports the theoretical results by (Lin, 2002; Facskó et al., 2008; Facskó et al., 2009). The distribution of TD normals for $\gamma>45^{\circ}$ is evenly distributed. We most often used ACE MAG measurements to determine TD normals in 2003, but had to mainly use Cluster FGM magnetic field data in 2007 because it was impossible to couple ACE and Cluster observations. The simulation was a better description of the events of 2006 than that of 2007. This turns out to be an advantage because the accuracy of $\gamma$ and $\Delta \Phi$ increased in 2006 and 2007. 


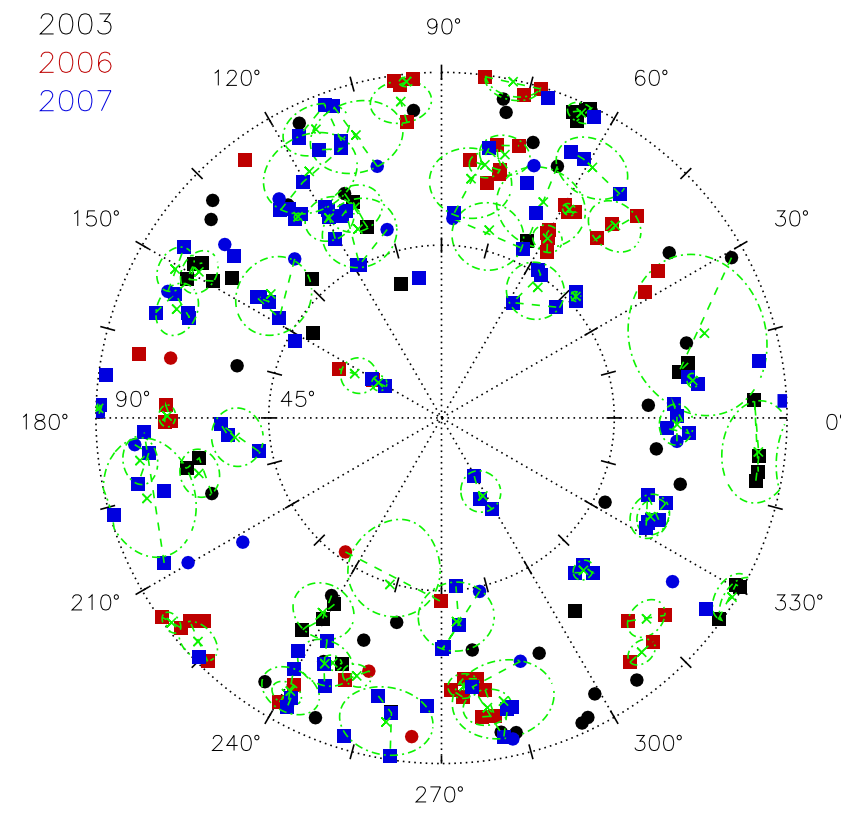

Fig. 4. Polar plot of the direction of the normal vectors of TDs. The azimuthal angle is measured between the GSE y direction and the projection of the normal vector onto the GSE yz plane. The distance from the center is the $\gamma$ angle as determined by the cross-product method. The TD normal vector is in a special polar coordinate system in which we measure the $\gamma$ angle from the center, and where the azimuth is the angle of GSE $y$ and the projection of normal vector to GSE yz plane. The regions surrounded by dashed lines are the projection of error cones around the average normal vector marked by "X". Circles and squares symbolize ACE and Cluster data, respectively. The black, red and blue symbols present events observed in 2003, 2006 and 2007, respectively.

\subsubsection{Estimations of HFA size}

Cluster satellites cross HFAs but the time length of the event holds no information about the real size of the phenomena because the boundaries of the cavity rim are not in pressure balance (Thomsen et al., 1986; Lucek et al., 2004) and the HFA also moves in the frame of the solar wind plasma. On the other hand, we have other valuable information: the time that the spacecraft spends inside the cavity gives a lower limit for the time of the existence of the HFA. One can calculate the error based on the measurements of four (or less) satellites. The size of the HFA must be estimated in another way.

1. HFAs, hot diamagnetic cavities, are created by particle beams accelerated by the supercritical bow shock. The beam shares its energy through electromagnetic ion-ion beam instability. In fact, this beam creates Alfvén waves and these waves carry away a larger part of the energy; only $2 / 3$ of the energy heats the plasma (Thomas and Brecht, 1988; Thomas, 1989). The propagation velocity of these waves does not exceed the Alfvén velocity so that twice the Alfvén speed multiplied by time of existence may give a rough estimate for the lower limit of the HFA size. Schwartz et al. (1985) determined the expansion speed of the cavity using ISEE-1 and ISEE-2 measurements, and the measured expansion speed was approximately the same as the estimated velocity.

2. HFAs are formed by the interaction of the bow shock and a tangential discontinuity. In many numerical simulations (Burgess and Schwartz, 1988; Lin, 2002; Omidi and Sibeck, 2007) and observations (Lucek et al., 2004) one can see that the HFA appears when the TD reaches the quasi-parallel region and remain while the TD sweeps the surface of the bow-show. We calculated the transit velocity of the tangential discontinuity on the surface of the bow shock using Schwartz et al. (2000)'s formula:

$\boldsymbol{V}_{\mathrm{tr}}=\frac{\boldsymbol{V}_{\mathrm{sw}} \boldsymbol{n}_{\mathrm{cs}}}{\sin ^{2} \theta_{\mathrm{cs}: \mathrm{bs}}}\left(\boldsymbol{n}_{\mathrm{cs}}-\cos \theta_{\mathrm{cs}: \mathrm{bs}} \boldsymbol{n}_{\mathrm{bs}}\right)$,

where $\boldsymbol{V}_{\text {tr }}$ is the transient velocity, $\boldsymbol{V}_{\mathrm{sw}}$ is the solar wind speed, $\boldsymbol{n}_{\mathrm{cs}}$ is the normal of the tangential discontinuity (current sheet), $\boldsymbol{n}_{\mathrm{bs}}$ is the normal of the bow shock, and $\theta_{\text {cs:bs }}$ is the angle between the two previously mentioned normals. The bow shock shape, position and normal were calculated by the model described in Peredo et al. (1995) as in the original paper which used ACE SWEPAM measurements. The solar wind vectors were determined by using Cluster CIS HIA measurements. This instrument operates only on Cluster SC1 and SC3. We obtained two estimates on the size of HFA. The obtained sizes are very similar after multiplying the velocity by the transition time of the spacecraft.

We estimated the size of HFAs and the errors based on the methods above. Each of them gives four results by four satellites. We took the average over the four points to be the size, with the standard deviation as the error. Unfortunately the CIS HIA aboard Cluster- 1 and Cluster- 3 provided unusually high temperatures close to the bow shock and so we used only the measurements of the ACE SWEPAM plasma instrument and the ACE MAG magnetometer to determine the properties of the plasma. For this reason only one size distribution from using the first method is given (Fig. 5). The average sizes and their errors are $(1.9 \pm 1.0) R_{\text {Earth }}$, (7.0 \pm 4.3$) R_{\text {Earth }}$ and (6.6 \pm 4.2$) R_{\text {Earth }}$, respectively. The first result confirms the predictions of the Lin's theory however the second result seems to be much higher. Most of the distribution functions of the second estimation shows a value of approximately $5 R_{\text {Earth }}$. The reason for this higher average is the "tail" of the distribution at larger sizes. Unfortunately this size estimation is very sensitive to the errors of the different normals and velocity vectors (see Eq. 1) and often gives a very large size. After comparing the size distributions of two methods on Fig. 5 one can see that most of their values 
do not differ by more then a factor of two. They are thus suitable for estimating the size of the phenomena. All side distributions are found to be very similar and the size-angle functions support the simulation results.

\subsubsection{Size-angle and size-speed scatter plots}

Size-angle relations were reported in Lin (2002). Furthermore we were informed about size-speed predictions (Y. Lin, personal communication, 2007).

Figure 6 show the size- $\gamma$ correlations. The error of the size was calculated by the method described by Sect. 3.1.2 and the error of the angles was estimated by the cross-product method: we calculated the direction for every single spacecraft, the average of these directions, and finally the error cone. The error of direction was not calculated where only one direction was obtained. It is very important to remark that the size depends not on one but three parameters. The size was plotted as a function of one parameter $(\gamma)$ while the speed and $\Delta \Phi$ values were fixed. In fact, fixing a parameter means fixed angle intervals because these were real measurements and not theoretical models. We fixed the speed in Alfén-Mach number in the simulation as well. We chose these $\Delta \Phi$ intervals because these contains those points which were simulated by Lin with $M_{\mathrm{A}}=5$ and $\Delta \Phi=80^{\circ}$. We obtained a maximum of the size- $\gamma$ scattered plot but not exactly at $\gamma=80^{\circ}$ in both cases as predicted (Lin, 2002). The other panels also support the theory since a maximum is visible on every panel. When we plotted all points we obtained a "cloud" of points with a maximum value.

Figure 7 presents the size- $\Delta \Phi$ functions where $\Delta \Phi$ is the change angle of magnetic field direction across the TD. The error of the size and angle were calculated the same way as at size- $\gamma$ functions. Here $\gamma$ and the solar wind speed were fixed and we used Alfvén Mach numbers. Here the bottom panels show the case studied in the simulation of Lin (2002). All panels show monotonically increasing size- $\Delta \Phi$ functions, confirming simulation results. We obtain a set of points a dense region that increases to the larger sizes.

In Fig. 8 the dependence of HFA size on velocity is visible in several fixed angle intervals. Solar wind speed was measured in Alfvén Mach number value. The size was estimated based on the Alfvén speed method (black) and by calculating the velocity of the intersection line of the TD and the bow shock (red). The angular dependence of size was studied in a fixed intervals around $\gamma=80^{\circ}$ and $\Delta \Phi=40^{\circ}$ angles and the size is the monotonically growing function of the Alfvén Mach number.

\subsection{Speed distributions}

We observed in our previous work (Kecskeméty et al., 2006) that the value of the solar wind speed is close to the average $\sim 400 \mathrm{~km} / \mathrm{s}$ but it is higher before HFAs are observed $(\sim 600 \mathrm{~km} / \mathrm{s})$. We have studied this point in more detail here.

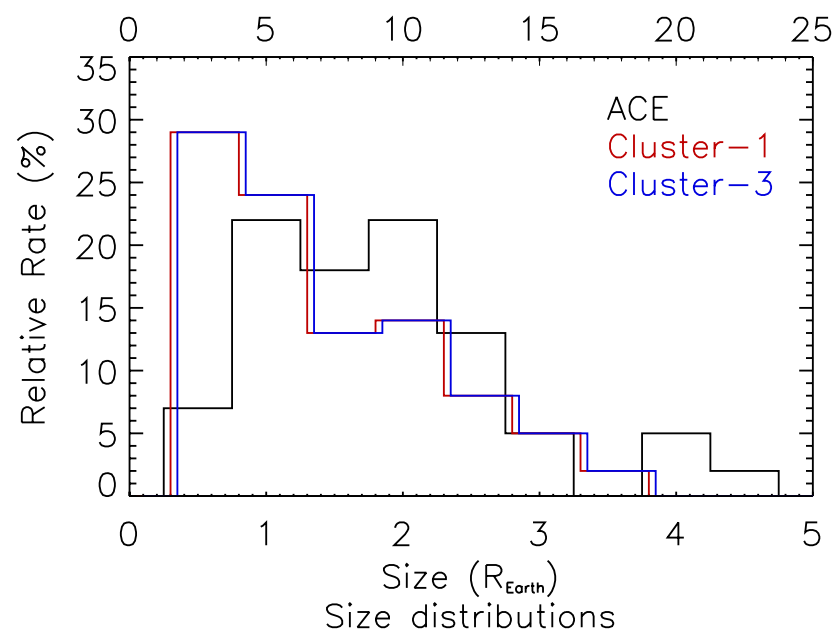

Fig. 5. The size distributions of HFAs estimated by Alfvén velocity and (solid line) the speed of the TD and bow shock intersection calculated by the solar wind measurements of Cluster-1 and -3 CIS HIA (red and blue line, scale drawn on top). The average sizes are (1.9 \pm 1.0$) R_{\text {Earth }},(7.0 \pm 4.3) R_{\text {Earth }}$ and (6.6 \pm 4.2$) R_{\text {Earth }}$, respectively.

The speed distributions were calculated here we used Cluster SC1 and SC3 CIS HIA; complemented by ACE SWEPAM data measured in longer time intervals to obtain better statistics. We recorded these solar wind speed values again when we used 5-10 min or even $30 \mathrm{~min}$ long intervals before the bow shock. We calculated the average, its scatter and plotted the distribution (Table 3, Fig. 9). We determined the time when the TD (which caused the HFA) crossed the position of ACE satellite and we determined the average solar wind parameters from ACE SWEPAM measurements. These results are in good agreement with earlier Cluster observations (Facskó et al., 2008; Facskó et al., 2009).

These speeds are obviously higher than the long-term averaged solar wind speed (Fig. 9a, b, d), and a peak appears on the distribution between $400 \mathrm{~km} / \mathrm{s}$ and $800 \mathrm{~km} / \mathrm{s}$ measured instead of the expected $400 \mathrm{~km} / \mathrm{s}$ or $800 \mathrm{~km} / \mathrm{s}$ peaks measured by Ulysses (McComas et al., 2003), but it is in question whether this difference is really significant. The average speed for the full-studied time period using ACE SWEPAM (Fig. 9e, black line) was $(546 \pm 97) \mathrm{km} / \mathrm{s}$ in 2003 . Actually, the solar wind speed was higher throughout the studied period in 2003 (Fig. 10). Measurements of ACE from 1998 to 2008 (Fig. 9e, green line) yielded $(498 \pm 101) \mathrm{km} / \mathrm{s}$ suggesting that during HFA formation the typical solar wind speed is higher than the average value by almost $200 \mathrm{~km} / \mathrm{s}$ than the average value. It seems that the presence of a fast solar wind is a necessary condition of the formation of HFAs. This is obvious when one looks at the bottom panel of Fig. 10, where we plotted the studied interval using $1 \mathrm{~h}$ averaged solar wind speed. The HFAs marked by vertical lines and their positions all appear in fast solar wind regimes. In fact almost all HFA 


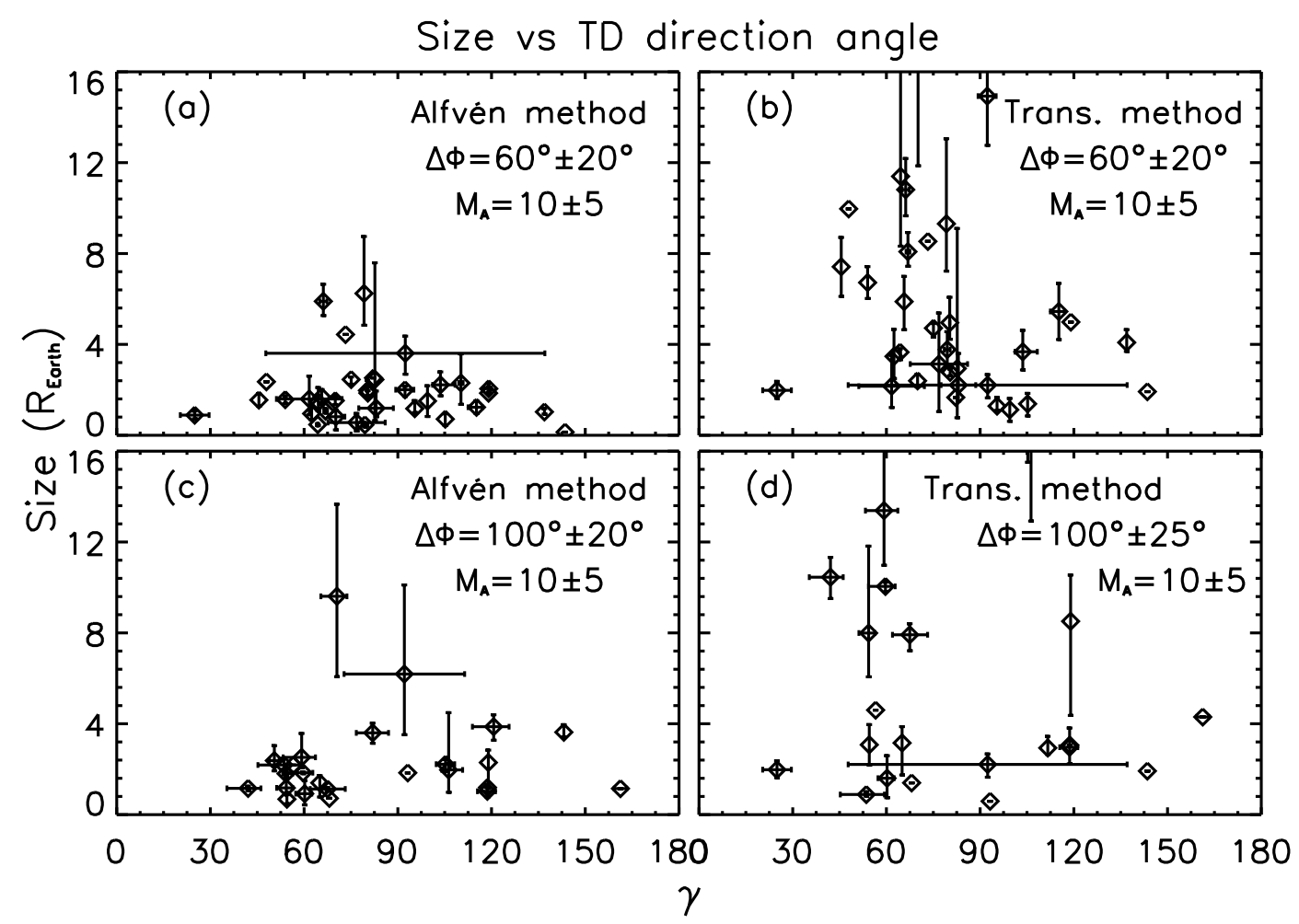

Fig. 6. The size- $\gamma$ functions based on the size estimation by Alfvén Mach velocity on the left and the transition speed on the right. The fixed solar wind speed was shown in Alfvén Mach number. (a) $\Delta \Phi=60^{\circ} \pm 20^{\circ}$ and $M_{\mathrm{A}}=10 \pm 5$, (b) $\Delta \Phi=60^{\circ} \pm 20^{\circ}$ and $M_{\mathrm{A}}=10 \pm 5$, (c) $\Delta \Phi=100^{\circ} \pm 20^{\circ}$ and $M_{\mathrm{A}}=10 \pm 5$, (d) $\Delta \Phi=100^{\circ} \pm 25^{\circ}$ and $M_{\mathrm{A}}=10 \pm 5$. All Alfvén Mach numbers were calculated from the actual Alfvén velocity.

Table 3. Solar wind speed, fast magnetosonic Mach number mean values, and their deviations measured by Cluster CIS and ACE SWEPAM. The last column gives the figure numbers shown on Fig. 9.

\begin{tabular}{rcccc}
\hline solar wind speed $(\mathrm{km} / \mathrm{s})$ & 2003 & 2006 & 2007 & Fig. \\
\hline during HFA formation by C1 & $680 \pm 86$ & $614 \pm 84$ & $613 \pm 80$ & $9 \mathrm{a}$ \\
by C3 & $671 \pm 92$ & $614 \pm 82$ & $613 \pm 78$ & $9 \mathrm{~b}$ \\
by ACE & $666 \pm 84$ & $626 \pm 85$ & $634 \pm 71$ & $9 \mathrm{~d}$ \\
$M_{\mathrm{f}}$ numbers by ACE & $8.2 \pm 1.2$ & $9.1 \pm 1.0$ & $9.9 \pm 1.1$ & $9 \mathrm{c}$ \\
between 1998-2003/2008 by ACE & $492 \pm 102$ & $498 \pm 101$ & $9 \mathrm{e}$ \\
$M_{\mathrm{f}}$ numbers by ACE & $5.5 \pm 1.4$ & $6.2 \pm 1.7$ & $9 \mathrm{f}$ \\
$\Delta M_{\mathrm{f}}$ & 2.7 & 2.9 & 3.7 & \\
\hline
\end{tabular}

events appeared in the same co-rotating region (Facskó et al., 2008; Facskó et al., 2009). The frequency of fast solar wind beams in the Ecliptic depends on the solar cycle. The frequency of HFAs is thus expected to depend on solar cycle. After processing the measurements in 2006 and 2007 this cannot be confirmed because the average number of HFAs is about 2 HFAs/day with large scatter $(2.2 \pm 1.2,2.5 \pm 1.4$ and $2.1 \pm 1.5$ in 2003, 2006 and 2007, respectively) so there is no significant difference during the different seasons. There were several longer HFA series in 2006 and 2007 but not in is 2003. The difference between the solar wind speeds were high $-\sim 130 \mathrm{~km} / \mathrm{s}-$ but not as high as in 2003 . Based on three years of measurements of we can conclude that the higher solar wind speed might be an important requirement for the HFA formation mechanism. We found only a few HFAs out of the fast solar wind co-rotating regions. 


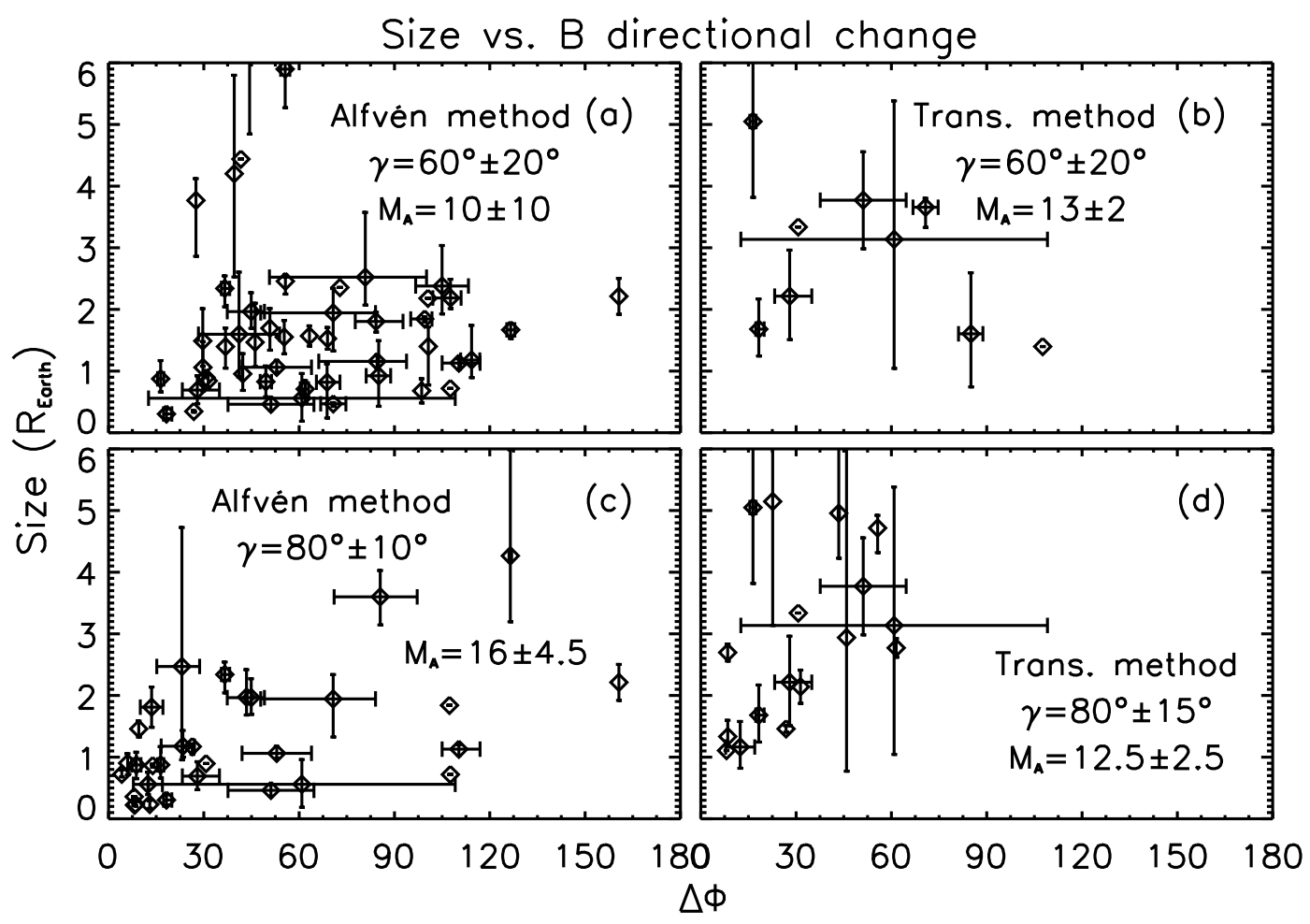

Fig. 7. The size- $\Delta \Phi$ functions based on the size estimation by Alfvén Mach velocity on the left and the transition speed on the right. The fixed solar wind speed was shown in Alfvén Mach number. (a) $\gamma=60^{\circ} \pm 20^{\circ}$ and $M_{\mathrm{A}}=10 \pm 10$, (b) $\gamma=60^{\circ} \pm 20^{\circ}$ and $M_{\mathrm{A}}=13 \pm 2$, (c) $\gamma=80^{\circ} \pm 10^{\circ}$ and $M_{\mathrm{A}}=16 \pm 4.5$, (d) $\gamma=80^{\circ} \pm 15^{\circ}$ and $M_{\mathrm{A}}=12.5 \pm 2.5$. All Alfvén Mach numbers were calculated from the actual Alfvén velocity.

Figure 9c shows a more unexpected result. The figure shows the distribution of the fast-magnetosonic Mach numbers during HFA formation. The Mach numbers are very high, with $M_{\mathrm{f}} \geq 6$ in 2003, this can also be observed in 2006 and 2007 where the difference between them is even greater. This is made more obvious if we compare this distribution to the distribution calculated by ACE SWEPAM and MAG measurements for the studied interval and all measurements of ACE (Fig. 9f). Both longer periods show that these high Mach numbers are very rare (Facskó et al., 2008). The HFAs are not only Earth-specific features (Øieroset et al., 2001). The Mach numbers are in general much larger in the outer Solar System, since the propagation speed of fast magnetosonic waves is lower due to the weaker magnetic field. This fact suggests that HFA events might be even more frequent at Saturn, for instance the other giant planets in the Solar System.

\subsection{Solar wind density and pressure}

Several HFA events are shown on Fig. 10 when the solar wind velocity is above average, but which do not have very large values. The higher solar wind velocity seems to be a necessary condition of forming HFAs so these exceptions look strange. We studied parameters, one of which was so-

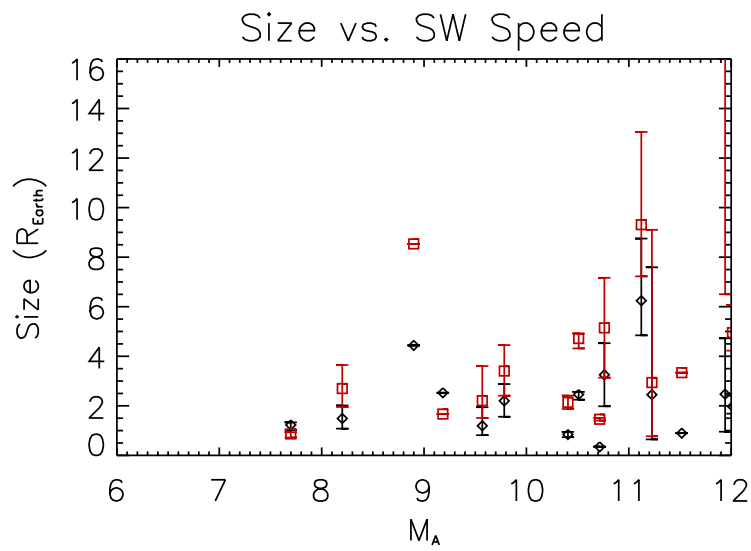

Fig. 8. The size-velocity functions with Alfvén velocity calculated using ACE and crossing time measured by Cluster. The sizes were calculated using the method based on Alfvén speed (black) and the transition speed (red). The fixed solar wind speed was measured in units of Alfvén Mach number. $\gamma=80^{\circ} \pm 10^{\circ}$ and $\Delta \Phi=40^{\circ} \pm 20^{\circ}$. All Alfvén Mach numbers were calculated from the actual Alfvén velocity.

lar wind particle density. (Fig. 11a). We noticed that the particle density is below the average during an HFA formation, at $3.6 \pm 1.4 \mathrm{~cm}^{-3}$ instead of the long-term average value 


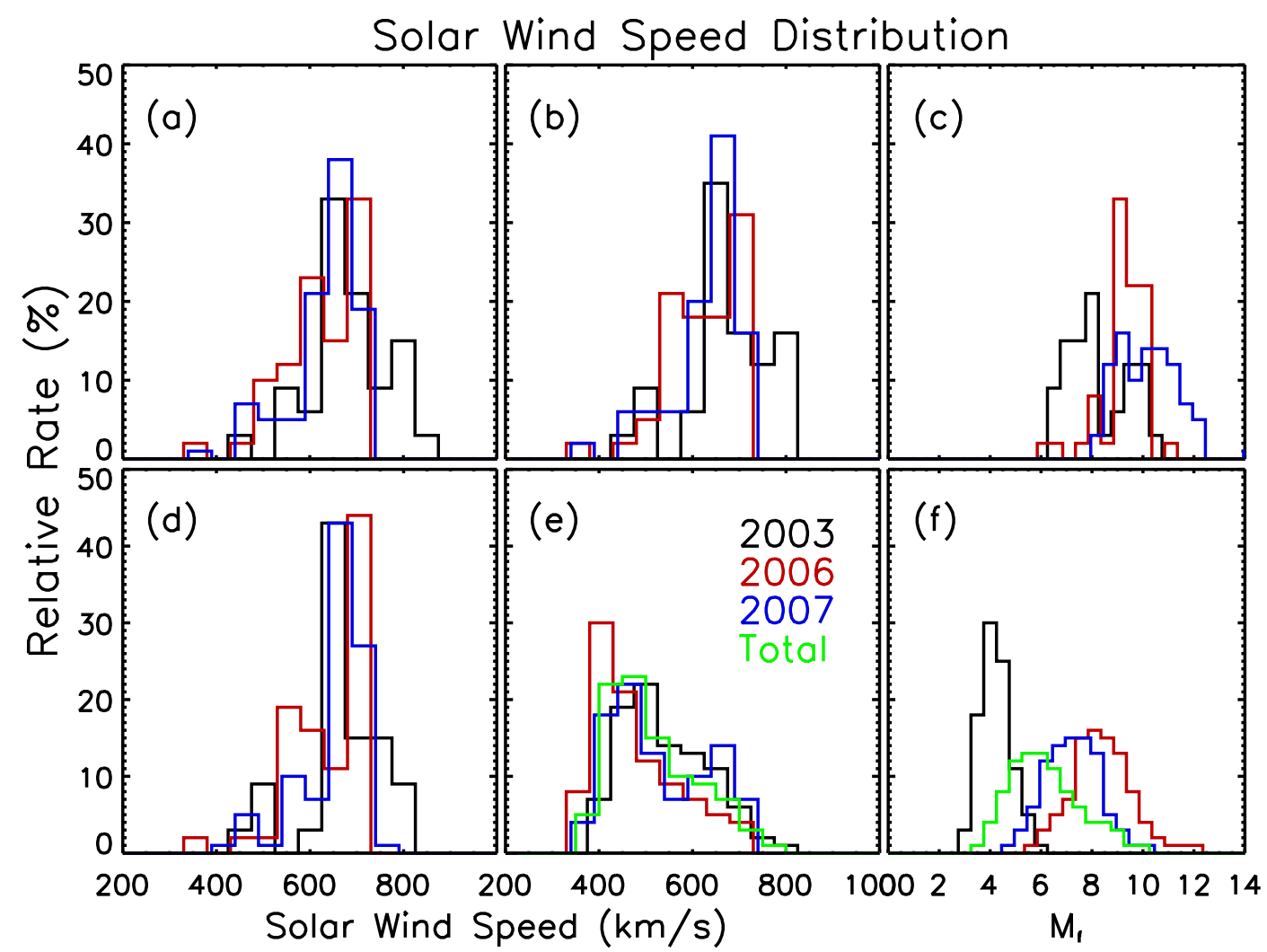

Fig. 9. Solar wind speed distribution measured by Cluster and ACE spacecraft. Black, red, blue and green refers to measurements in 2003, 2006, 2007 and 1998-2008, respectively. The figure shows the solar wind speed distribution measured by (a) Cluster-1 CIS HIA during HFA formation. (b) by Cluster-3 CIS HIA, and (d) by ACE SWEPAM; it also shows. Fast magnetosonic Mach number distribution calculated using ACE MAG and SWEPAM data during HFA formation (c), solar wind speed distribution measured by ACE SWEPAM from February to April 2003, December 2005-April 2006 and January-April 2007 and 1998-2008 (e), and fast-magnetosonic Mach-number distribution (f).

of $6.9 \pm 4.2 \mathrm{~cm}^{-3}$ (based on the ACE SWEPAM $1 \mathrm{~h}$ average data series measured between 1998 and 2008). This observation is not surprising since the solar wind pressure is approximately constant. Thus, if the solar wind velocity is higher, the density is expected to be lower.

The other studied parameter was the solar wind pressure. We also calculated distribution function, which suggested lower pressure during HFA formation than the average of all measurements of ACE from 1998 to 2008. It was $1.7 \pm 0.8 \mathrm{nP}$ instead of the $1.9 \pm 1.2 \mathrm{nPa}$ (Fig. 11b). In our opinion this difference is not significant. Unfortunately the high solar wind pressure does not seem to be a condition of HFA formation in the case of those few events when the solar wind speed is not too large.

\subsection{Schwartz et al.'s condition}

We have checked whether the Schwartz et al. (2000) condition is valid for our HFA events. The above discussed analysis of HFA events in the spring 2003, 2006 and 2007 seasons confirmed and extended our earlier results based on the study of HFA events in spring 2003. These showed that higher so- lar wind speed is an important condition of HFA formation. This feature restricts the formula of Schwartz et al. (2000) because the TD must slowly sweep the bow shock which is possible for only a very limited geometrical condition. Besides of these limitations our events also confirm the following results:

$\left|\frac{V_{\mathrm{tr}}}{V_{\mathrm{g}}}\right|=\frac{\cos \theta_{\mathrm{cs}: \mathrm{sw}}}{2 \cos \theta_{\mathrm{bs}: \mathrm{sw}} \sin \theta_{\mathrm{B}_{\mathrm{n}}} \sin \theta_{\mathrm{cs}: \mathrm{bs}}}<1$,

where $V_{\text {tr }}$ is the transit velocity of the current sheet along the bow shock, $V_{\mathrm{g}}$ is the gyration speed, $\theta_{\mathrm{cs}: s w}, \theta_{\mathrm{bs}: s w}$ and $\theta_{\text {cs:bs }}$ are the angles between the discontinuity normal, solar wind velocity and the bow shock, and finally $\theta_{\mathrm{B}_{n}}$ is the angle between the magnetic field and bow shock normal. The necessary vectors were calculated using Cluster SC1 and SC3 CIS HIA measurements (Fig. 12). We found that the transition speed is most often as low as expected by the formula of Schwartz et al. (2000). This formula usually gives a value of less than 1 one during HFA formation. Here the formula often gives a greater value than one; however, this study also confirms that HFA formation also depends on the geometry of the shock, the discontinuity, and the solar wind velocity. 

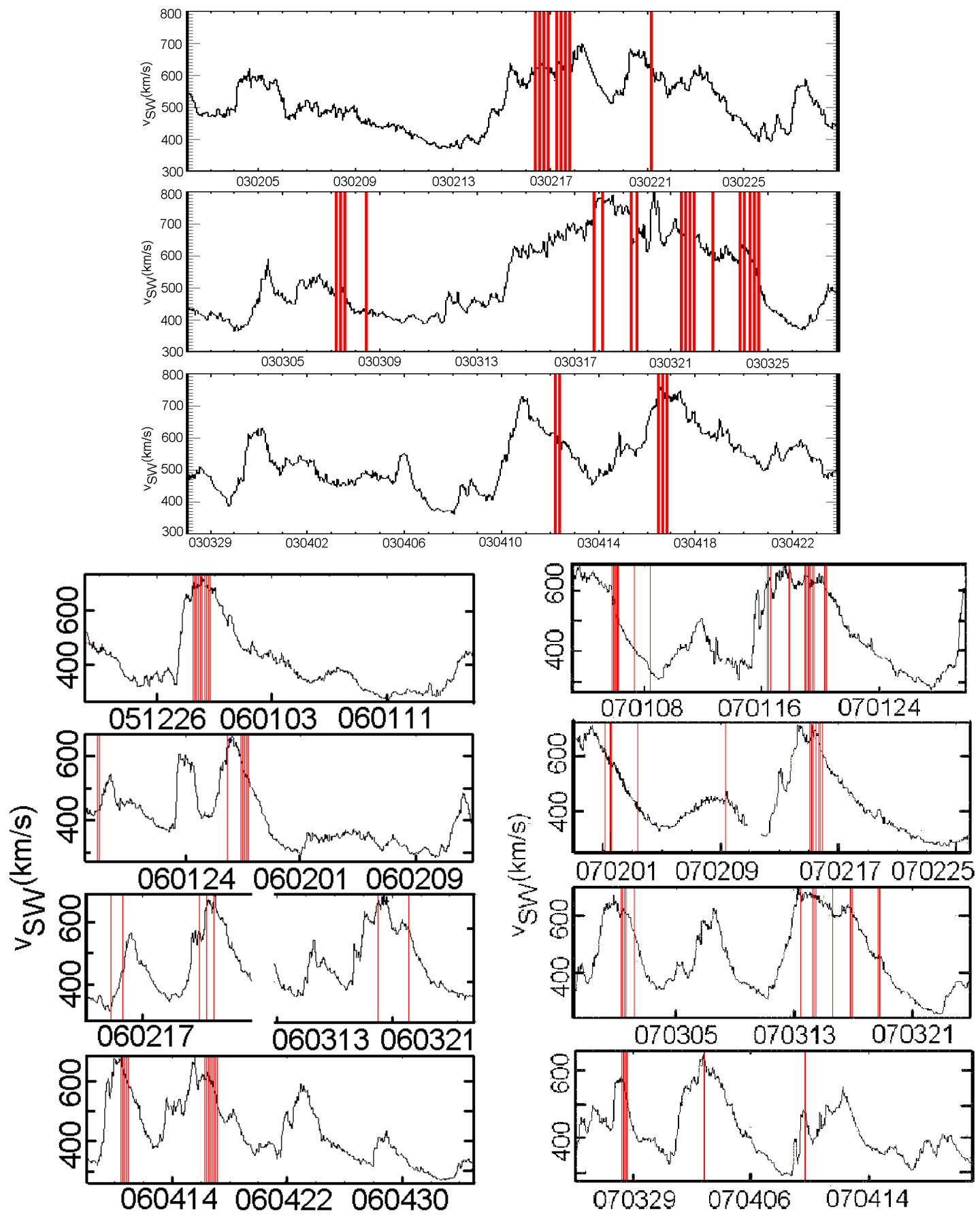

Fig. 10. $1 \mathrm{~h}$ averaged solar wind speed; the vertical red lines give the time of HFAs. The top, bottom left and right figures were measured by ACE SWEPAM instrument in 2003, 2006 and 2007, respectively. The connection between the fast solar wind regions and the HFAs is evident.

\section{Discussion}

Our resulting value of size estimation, the shape of sizeangle and size-velocity distributions, as well as the function of $\Delta \Phi$ and $\gamma$, confirm previous predictions of numerical simulations. The large number of events, as well as the higher solar wind speed and Mach number are new results although Koval et al. (2005) had made similar observations using INTERBALL-1 and MAGION-4 spacecraft. (That study was performed using magnetosheath observation instead of upstream measurements.) All our observations agree well with current theories and simulations. 


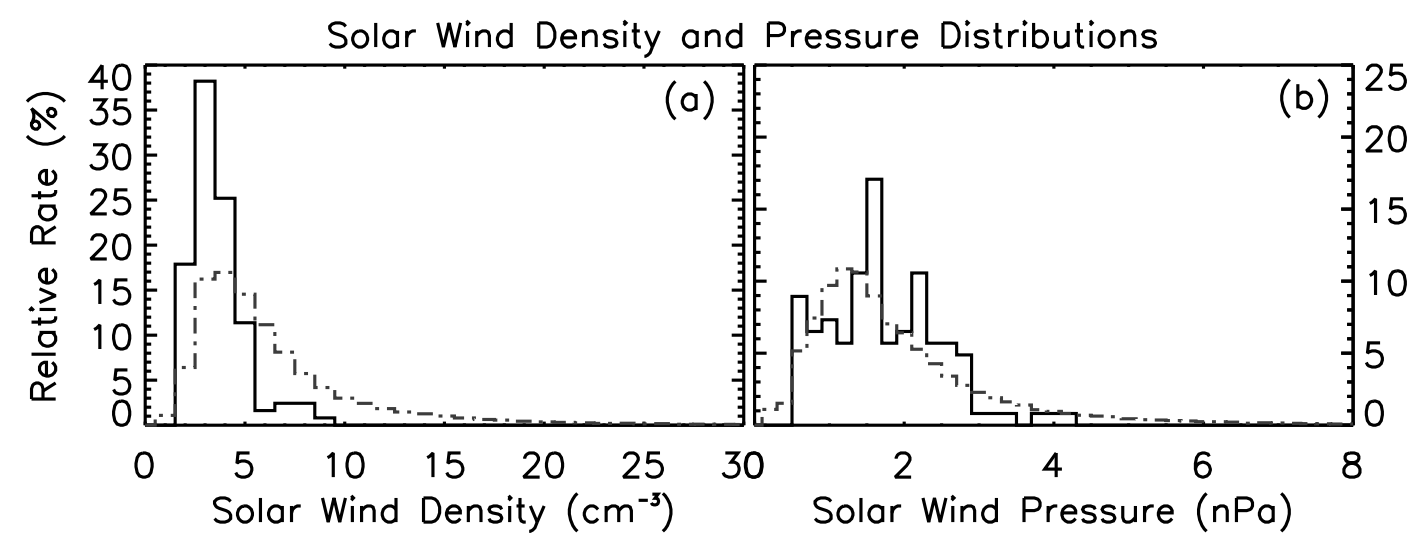

Fig. 11. (a) Solar wind particle density distribution during HFA events (dash-dotted line) using ACE SWEPAM measurements from 1998 to 2008 (solid line). (b) Solar wind pressure in the same time intervals.

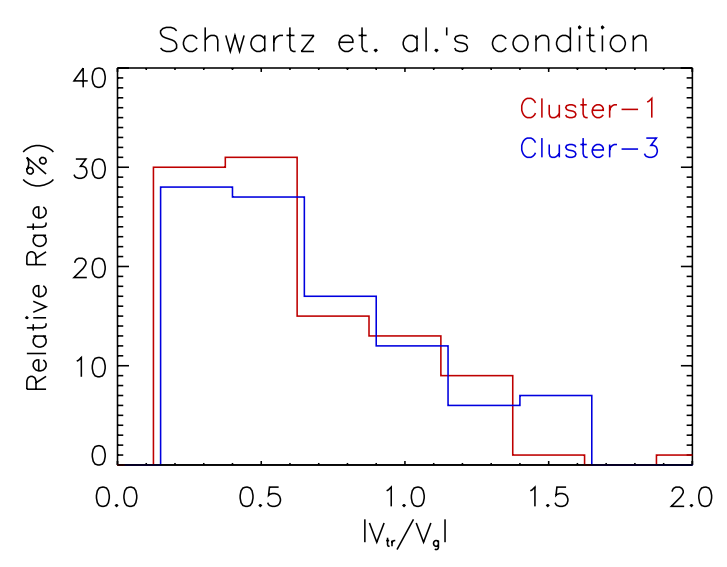

Fig. 12. The distribution of the rate given by Eq. (2). We use both Cluster SC1 and SC3 CIS HIA measurements to determine the necessary vectors in the formula. The red and blue lines show the distribution based on Cluster-1 and -3 measurements.

The high solar wind velocity as an essential condition is logical and acceptable because particles of the beam which form the HFA are accelerated at the supercritical bow shock. Here, the particles are forced to return to the foreshock region approximately with solar wind speed, but antiparalel to solar wind velocity (Gosling and Robson, 1985; Kennel et al., 1985; Scholer et al., 1993; Tanaka et al., 1983; Quest, 1989). This process causes the heating of the region and the energy dissipation of the flow, and forms the beam which creates the HFA. The higher the speed of the solar wind, the higher the energy of the reflected beams. Moreover, analytical calculations by Németh (2007) (which study the possible particle trajectories of trapped ions in the vicinity of shock-discontinuity crossings) suggest high solar wind speed as a favorable condition of particle reflection. Unfortunately no numerical simulation thus for can predict this condition, probably because these simulations are constrained into 2 spatial dimensions. 3-D hybrid simulations may be able to predict the high solar wind speed condition.

The $\gamma$ distribution and the size maxima of size- $\gamma$ functions (Lucek et al., 2004; Schwartz et al., 2000) are explained as follows: acceleration needs time and the TD must approach the bow shock. If the angle is large then it approaches slower and there is more time for acceleration. Beyond at given angle particles do not bounce back and nothing forms. The situation is different in the case of growing size- $\Delta \Phi$ functions. Y. Lin (personal communication, 2007) suggests that the electric field depends on this angle, so larger $\Delta \Phi$ generates larger electric field which focuses particles to the TD. It is well known that the acceleration happens between the TD and the quasi-parallel shock. When the TD reaches the quasiparallel region of the bow shock or when the TD changes the magnetic field direction, the particles - which form the beam - can escape from the trap, which gives rise to the phenomenon. Larger $\Delta \Phi$ causes longer acceleration time, which can explain the growing size- $\Delta \Phi$ functions.

The reason of the growing size-speed function can be the following: the beam that creates the HFAs is accelerated at the supercritical bow shock. This result is not surprising because their acceleration depends on the bow shock structure. A small amount of particles turns back and enters the region in front of the bow shock, the foreshock region or the region between the bow shock and the TD. TD occurs when the HFA is formed. The higher the velocity of the solar wind, the higher the speed of particles and size of the phenomenon. This trend can be seen on the Fig. 8, however it is not very obvious.

\section{Summary and conclusions}

Earlier we showed that HFAs are not as rare a phenomenon as it was a thought prior to Cluster (Kecskeméty et al., 2006). If a TD appears and the spacecraft are in the right position then the event can be observed with high probability if several 
special conditions are fulfilled. The numerous new HFA observations also confirm this opinion.

1. The most important condition is the larger solar wind velocity, which is typically much higher than the average speed. The differences were approximately $160 \mathrm{~km} / \mathrm{s}$ in 2003, and approximately $130 \mathrm{~km} / \mathrm{s}$ in 2006 and 2007.

2. The high fast magnetosonic Mach number is also a preferable condition for HFA formation. No events were found below $M_{\mathrm{f}}=6$ in 2003, and this limit increased in 2006 and 2007.

3. The pressure is irrelevant with respect to HFA formation. The solar wind particle density before the HFA events is lower than the average value of the solar wind density.

4. The angle between the TD normal $(\gamma)$ and Earth-Sun direction must be greater than $45^{\circ}$. Very few events were observed with $\gamma<45^{\circ}$.

5. The directional change of magnetic field within the TD $(\Delta \Phi)$ must be large. The average value was approximately $70^{\circ}$ based on 124 events.

6. Our size estimations do not contradict previous simulation results. We estimated $2-3 R_{\text {Earth }}$ size using one method; the other method gave larger sizes in the range of $1 R_{\text {Earth }}$. The differences can be explained with the high sensitivity of the methods to the accuracy of the measurements.

7. The size-angle and size-speed plots of Lin (2002) were reproduced in good agreement with the predictions.

8. The conditions were mostly quasi-parallel during HFA formation, which is unexpected because the HFA determination decreases the number of quasi-parallel cases. So our HFA observations confirm the previous simulation result of Omidi and Sibeck (2007) and showed that HFAs appear where the quasi-perpendicular condition turns to quasi-parallel. Furthermore, the particles of the beam escape in the quasi-parallel part of the bow shock.

9. We also confirmed the suggestion of Schwartz et al. (2000), namely that the transition velocity of the HFA at the bow shock must be slow. Furthermore, our new result does not contradict to the formula presented in that paper (Eq. 2).

We have determined the typical size of HFAs in two different ways. The number of HFAs does not depend on solar activity, only on the time of periods when the solar wind velocity is high. We compared within the theoretical predictions and proved that they are correct in 2003, 2006 and 2007 when the Cluster fleet separation was large. All observations agree well with current theories and confirm the simulation results. We also publish here the detected events and their parameters. We hope they will be used to further studies, for example, THEMIS-Cluster multi-multispacecraft observations or further statistical investigations beyond and inside the bow shock.

The reason why the high solar wind velocity is necessary for HFA formation was not explained in detail. Further probably 3-D hybrid - simulations are necessary to clarify the theoretical background of this behavior.

Acknowledgements. The authors thank the ACE MAG and SWEPAM working teams for the magnetic field and plasma data; furthermore the authors are also very grateful to Mariella Tátrallyay for providing high resolution Cluster FGM data files. The present work was supported by the OTKA grant K75640 of the Hungarian Scientific Research Fund. Gábor Facskó thanks Pierrette Decreau and Robert Ferdman for their help in improving the English of this paper.

Topical Editor R. Nakamura thanks N. Omidi and another anonymous referee for their help in evaluating this paper.

\section{References}

Balogh, A., Carr, C. M., Acuña, M. H., Dunlop, M. W., Beek, T. J., Brown, P., Fornacon, K.-H., Georgescu, E., Glassmeier, K.H., Harris, J., Musmann, G., Oddy, T., and Schwingenschuh, K.: The Cluster Magnetic Field Investigation: overview of in-flight performance and initial results, Ann. Geophys., 19, 1207-1217, 2001, http://www.ann-geophys.net/19/1207/2001/.

Burgess, D. and Schwartz, S. J.: Colliding plasma structures - Current sheet and perpendicular shock, J. Geophys. Res., 93, 11327 11340, 1988.

CIS Team: CAVEATS for the Data supplied by the CIS Experiment Onboard the Cluster Spacecraft, web page, 1997-present.

Facskó, G., Tátrallyay, M., Erdős, G., and Dandouras, I.: Cluster hot flow anomaly observations during solar cycle minimum, in: Proceedings of the 15th Cluster Workshop \& Cluster Active Archive School, Springer Verlag, in press, 2009.

Facskó, G., Kecskeméty, K., Erdős, G., Tátrallyay, M., Daly, P. W., and Dandouras, I.: A statistical study of hot flow anomalies using Cluster data, Adv. Space Res., 41, 1286-1291, doi:10.1016/j.asr. 2008.02.005, 2008.

Gosling, J. T. and Robson, A. E.: Ion reflection, gyration, and dissipation at supercritical shocks, Washington D.C. American Geophysical Union Geophysical Monograph Series, 35, 141-152, 1985.

Kecskeméty, K., Erdős, G., Facskó, G., Tátrallyay, M., Dandouras, I., Daly, P., and Kudela, K.: Distributions of suprathermal ions near hot flow anomalies observed by RAPID aboard Cluster, Adv. Space Res., 38, 1587-1594, doi:10.1016/j.asr.2005.09.027, 2006.

Kennel, C. F., Edmiston, J. P., and Hada, T.: A quarter century of collisionless shock research, Washington D.C. American Geophysical Union Geophysical Monograph Series, 34, 1-36, 1985.

Knetter, T., Neubauer, F. M., Horbury, T., and Balogh, A.: Fourpoint discontinuity observations using Cluster magnetic field 
data: A statistical survey, J. Geophys. Res., 109, 6102, doi: 10.1029/2003JA010099, 2004.

Koval, A., Šafránková, J., and Němeček, Z.: A study of particle flows in hot flow anomalies, Planet. Space Sci., 53, 41-52, doi: 10.1016/j.pss.2004.09.027, 2005.

Lin, Y.: Global hybrid simulation of hot flow anomalies near the bow shock and in the magnetosheath, Planet. Space Sci., 50, 577-591, 2002.

Lucek, E. A., Horbury, T. S., Balogh, A., Dandouras, I., and Rème, H.: Cluster observations of hot flow anomalies, J. Geophys. Res., 109, 6207, doi:10.1029/2003JA010016, 2004.

McComas, D. J., Bame, S. J., Barker, P., Feldman, W. C., Phillips, J. L., Riley, P., and Griffee, J. W.: Solar Wind Electron Proton Alpha Monitor (SWEPAM) for the Advanced Composition Explorer, Space Sci. Rev., 86, 563-612, doi:10.1023/A: 1005040232597, 1998.

McComas, D. J., Elliott, H. A., Schwadron, N. A., Gosling, J. T., Skoug, R. M., and Goldstein, B. E.: The three-dimensional solar wind around solar maximum, Geophys. Res. Lett., 30, 24-1, doi: 10.1029/2003GL017136, 2003.

Németh, Z.: Particle acceleration at the interaction of shocks and discontinuities, in: Proceedings of the 30th International Cosmic Ray Conference, 2007.

Øieroset, M., Mitchell, D. L., Phan, T. D., Lin, R. P., and Acuña, M. H.: Hot diamagnetic cavities upstream of the Martian bow shock, Geophys. Res. Lett., 28, 887-890, doi:10.1029/ 2000GL012289, 2001.

Omidi, N. and Sibeck, D. G.: Formation of hot flow anomalies and solitary shocks, J. Geophys. Res. (Space Physics), 112, 1203, doi:10.1029/2006JA011663, 2007.

Onsager, T. G., Winske, D., and Thomsen, M. F.: Interaction of a finite-length ion beam with a background plasma - Reflected ions at the quasi-parallel bow shock, J. Geophys. Res., 96, 17751788, 1991.

Peredo, M., Slavin, J. A., Mazur, E., and Curtis, S. A.: Threedimensional position and shape of the bow shock and their variation with Alfvenic, sonic and magnetosonic Mach numbers and interplanetary magnetic field orientation, J. Geophys. Res., 100, 7907-7916, 1995.

Quest, K.: Hybrid Simulation, in: Tutorial Courses: Third International School for Space Simulation, Toulouse, France, edited by: Lembege, B., Eastwood, J., and Nepadues, E., p. 177, 1989.

Rème, H., Aoustin, C., Bosqued, J. M., Dandouras, I., Lavraud, B., Sauvaud, J. A., Barthe, A., Bouyssou, J., Camus, Th., CoeurJoly, O., Cros, A., Cuvilo, J., Ducay, F., Garbarowitz, Y., Medale, J. L., Penou, E., Perrier, H., Romefort, D., Rouzaud, J., Vallat, C., Alcaydé, D., Jacquey, C., Mazelle, C., d’Uston, C., Möbius, E., Kistler, L. M., Crocker, K., Granoff, M., Mouikis, C., Popecki, M., Vosbury, M., Klecker, B., Hovestadt, D., Kucharek, H., Kuenneth, E., Paschmann, G., Scholer, M., Sckopke, N., Seidenschwang, E., Carlson, C. W., Curtis, D. W., Ingraham, C., Lin, R. P., McFadden, J. P., Parks, G. K., Phan, T., Formisano, V., Amata, E., Bavassano-Cattaneo, M. B., Baldetti, P., Bruno, R., Chionchio, G., Di Lellis, A., Marcucci, M. F., Pallocchia, G., Korth, A., Daly, P. W., Graeve, B., Rosenbauer, H., Vasyliunas, V., McCarthy, M., Wilber, M., Eliasson, L., Lundin, R., Olsen, S., Shelley, E. G., Fuselier, S., Ghielmetti, A. G., Lennartsson, W., Escoubet, C. P., Balsiger, H., Friedel, R., Cao, J.-B., Kovrazhkin, R. A., Papamastorakis, I., Pellat, R., Scudder, J., and Sonnerup, B.:
First multispacecraft ion measurements in and near the Earth's magnetosphere with the identical Cluster ion spectrometry (CIS) experiment, Ann. Geophys., 19, 1303-1354, 2001, http://www.ann-geophys.net/19/1303/2001/.

Scholer, M., Fujimoto, M., and Kucharek, H.: Two-dimensional simulations of supercritical quasi-parallel shocks: upstream waves, downstream waves, and shock re-formation, J. Geophys. Res., 98, 18971-18984, 1993.

Schwartz, S. J. and Burgess, D.: Quasi-parallel shocks - A patchwork of three-dimensional structures, Geophys. Res. Lett., 18, 373-376, 1991.

Schwartz, S. J., Chaloner, C. P., Hall, D. S., Christiansen, P. J., and Johnstones, A. D.: An active current sheet in the solar wind, Nature, 318, 269-271, 1985.

Schwartz, S. J., Paschmann, G., Sckopke, N., Bauer, T. M., Dunlop, M., Fazakerley, A. N., and Thomsen, M. F.: Conditions for the formation of hot flow anomalies at Earth's bow shock, J. Geophys. Res., 105, 12639-12650, doi:10.1029/1999JA000320, 2000.

Sibeck, D. G., Lopez, R. E., and Roelof, E. C.: Solar wind control of the magnetopause shape, location, and motion, J. Geophys. Res., 96, 5489-5495, 1991.

Sibeck, D. G., Borodkova, N. L., Schwartz, S. J., Owen, C. J., Kessel, R., Kokubun, S., Lepping, R. P., Lin, R., Liou, K., Lühr, H., McEntire, R. W., Meng, C.-I., Mukai, T., Nemecek, Z., Parks, G., Phan, T. D., Romanov, S. A., Safrankova, J., Sauvaud, J.-A., Singer, H. J., Solovyev, S. I., Szabo, A., Takahashi, K., Williams, D. J., Yumoto, K., and Zastenker, G. N.: Comprehensive study of the magnetospheric response to a hot flow anomaly, J. Geophys. Res., 104, 4577-4594, doi:10.1029/1998JA900021, 1999.

Sibeck, D. G., Phan, T.-D., Lin, R., Lepping, R. P., and Szabo, A.: Wind observations of foreshock cavities: A case study, J. Geophys. Res., 107, 4-1, doi:10.1029/2001JA007539, 2002.

Smith, C. W., L'Heureux, J., Ness, N. F., Acuña, M. H., Burlaga, L. F., and Scheifele, J.: The ACE Magnetic Fields Experiment, Space Sci. Rev., 86, 613-632, doi:10.1023/A:1005092216668, 1998.

Tanaka, M., Goodrich, C. C., Winske, D., and Papadopoulos, K.: A source of the backstreaming ion beams in the foreshock region, J. Geophys. Res., 88, 3046-3054, 1983.

Thomas, V. A.: Three-dimensional simulation of diamagnetic cavity formation by a finite-size plasma beam, J. Geophys. Res., 94, 13579-13583, 1989.

Thomas, V. A. and Brecht, S. H.: Evolution of diamagnetic cavities in the solar wind, J. Geophys. Res., 93, 11341-11353, 1988.

Thomas, V. A., Winske, D., Thomsen, M. F., and Onsager, T. G.: Hybrid simulation of the formation of a hot flow anomaly, J. Geophys. Res., 96, 11625-11632, 1991.

Thomsen, M. F., Gosling, J. T., Fuselier, S. A., Bame, S. J., and Russell, C. T.: Hot, diamagnetic cavities upstream from the earth's bow shock, J. Geophys. Res., 91, 2961-2973, 1986.

Thomsen, M. F., , Thomas, V. A., Winske, D., Gosling, J. T., Farris, M. H., and Russell, C. T.: Observational Test of Hot Flow Anomaly Formation by the Interaction of a Magnetic Discontinuity With the Bow Shock, J. Geophys. Res., 98, 15319-15330, 1993.

Tsyganenko, N. A.: Modeling the Earth's magnetospheric magnetic field confined within a realistic magnetopause, J. Geophys. Res., 100, 5599-5612, 1995. 